\title{
Combined effects of weight change trajectories and eating behaviors on childhood adiposity status: A birth cohort study
}

DOI:

10.1016/j.appet.2021.105174

\section{Document Version}

Accepted author manuscript

Link to publication record in Manchester Research Explorer

Citation for published version (APA):

Lin, Q., Jiang, Y., Wang, G., Sun, W., Dong, S., Deng, Y., Meng, M., Zhu, Q., Mei, H., Zhou, Y., Zhang, J., Clayton, P. E., Spruyt, K., \& Jiang, F. (2021). Combined effects of weight change trajectories and eating behaviors on childhood adiposity status: A birth cohort study. Appetite, 162, [105174].

https://doi.org/10.1016/j.appet.2021.105174

\section{Published in:}

Appetite

\section{Citing this paper}

Please note that where the full-text provided on Manchester Research Explorer is the Author Accepted Manuscript or Proof version this may differ from the final Published version. If citing, it is advised that you check and use the publisher's definitive version.

\section{General rights}

Copyright and moral rights for the publications made accessible in the Research Explorer are retained by the authors and/or other copyright owners and it is a condition of accessing publications that users recognise and abide by the legal requirements associated with these rights.

\section{Takedown policy}

If you believe that this document breaches copyright please refer to the University of Manchester's Takedown Procedures [http://man.ac.uk/04Y6Bo] or contact uml.scholarlycommunications@manchester.ac.uk providing relevant details, so we can investigate your claim.

\section{OPEN ACCESS}


Combined effects of weight change trajectories and eating behaviors on childhood adiposity status: A birth cohort study

Qingmin $\operatorname{Lin}^{1,2,3}$, Yanrui Jiang ${ }^{1,2,3}$, Guanghai Wang ${ }^{1,2,3}$, Wanqi Sun ${ }^{1,2,3}$, Shumei Dong ${ }^{1,2,3}$, Yujiao Deng ${ }^{1,2,3}$, Min Meng ${ }^{1,2,3}$, Qi Zhu ${ }^{1,2,3}$, Hao Mei ${ }^{2,4}$, Yingchun Zhou ${ }^{5}$, Jun Zhang ${ }^{3,6}$, Peter E. Clayton ${ }^{7}$, Karen Spruyt ${ }^{1,8}$, Fan Jiang ${ }^{1,2,3 \#}$

Qingmin Lin: qm12014@sjtu.edu.cn; Yanrui Jiang: yanrui1027@126.com; Guanghai Wang: wang-guanghai@163.com; Wanqi Sun: yiyunsays@hotmail.com; Shumei Dong: shumeidong1217@163.com; Yujiao Deng: yujiaodeng@126.com; Min Meng: mengmin0219@163.com; Qi Zhu: zhu_qi_yuland@163.com; Hao Mei: meihao@uw.edu; Yingchun Zhou: yczhou@stat.ecnu.edu.cn; Jun Zhang: junjimzhang@sina.com; Peter E. Clayton: peter.clayton@manchester.ac.uk; Karen Spruyt: spruytsleep@gmail.com; Fan Jiang: fanjiang@shsmu.edu.cn

\section{Affiliations:}

${ }^{1}$ Department of Developmental and Behavioral Pediatrics, Shanghai Children's Medical Center, Shanghai Jiao Tong University School of Medicine, Shanghai 200127, China.

${ }^{2}$ Pediatric Translational Medicine Institution, Shanghai Children's Medical Center, Shanghai Jiao Tong University School of Medicine, Shanghai 200127, China.

${ }^{3}$ MOE-Shanghai Key Laboratory of Children's Environmental Health, Xinhua Hospital, Shanghai Jiao Tong University School of Medicine, Shanghai 200092, China.

${ }^{4}$ Department of Data Science, School of Population Health, University of Mississippi Medical Center, Jackson, MS 39216, USA.

${ }^{5}$ KLATASDS-MOE, School of Statistics, East China Normal University, Shanghai 200062, China.

${ }^{6}$ School of Public Health, Shanghai Jiao Tong University, Shanghai 200025, China.

${ }^{7}$ Division of Developmental Biology and Medicine, Faculty of Biology, Medicine and Health, 
2 9PL, United Kingdom.

$3 \quad{ }^{8}$ INSERM, University Claude Bernard, School of Medicine, Lyon, France.

4

$5{ }^{\#}$ Corresponding Author: Fan Jiang, Department of Developmental and Behavioral Pediatrics,

6 Pediatric Translational Medicine Institution, Shanghai Children's Medical Center, Shanghai Jiao

7 Tong University School of Medicine, 1678 Dongfang Rd. Shanghai 200127, China. Tel: 86-21-

$8 \quad$ 38626012; Fax: 86-21-58706129; fanjiang@,shsmu.edu.cn

9 Declarations of interest: None 
1 Abbreviations

2 BMI: body mass index;

3 CI: confidence interval;

4 EF: enjoyment of food;

5 FF: food fussiness;

$6 \quad$ FR: food responsiveness;

$7 \quad$ SR: satiety responsiveness;

8 WHtR: waist-to-height ratio;

9 WAZ: weight-for-age z-score;

10 WAZ-change: the change of weight-for-age z-score 


\section{Introduction}

Over the last 40 years, overweight/obesity has become an epidemic ${ }^{[1]}$. When excess weight is accrued in early life, it can be persistent and is difficult to reverse ${ }^{[2]}$, predisposing children to a myriad of short- and long-term adverse physical and mental health outcomes ${ }^{[3,4]}$. In a cohort of 292,827 individuals, it was demonstrated that above average body mass index (BMI) in childhood, even at levels far below current overweight/obesity classifications, was associated with increased risk of lifelong health problems such as type 2 diabetes ${ }^{[5]}$. The prevention and reduction of childhood overweight/obesity or even higher BMI level than average may thus have the potential to realize substantial health benefits ${ }^{[6]}$. Strategies to maintain the weight of a growing child are likely to be much easier than encouraging a child with obesity to lose weight ${ }^{[7]}$. Therefore, identifying the critical periods and the risk factors for the occurrence and development of overweight/obesity early in life is the first and most important step for formulating preventive strategies.

Research suggests that the path to overweight/obesity is established very early in life ${ }^{[8]}$. Two systematic reviews (including one meta-analysis) have demonstrated that rapid weight change in infancy, commonly defined as a change of weight-for-age z-score (WAZ-change) greater than 0.67 over two time points, is associated with later life excessive body weight ${ }^{[9,10]}$. As several studies only investigated one infancy WAZ-change (e.g. between $0-6$ months $^{[11]}$, 0-1 year, and $0-2$ years $^{[10]}$ ), it was not possible to determine the "exact critical age window" in which the greatest WAZ-change occurred. Furthermore, a recent study identified distinct BMI-change trajectories from birth to age 14 years old that can provide guidance to design interventions targeting specific populations and specific ages, however, it did not capture the growth patterns in early life ${ }^{[12]}$. Whether a range of WAZ-change trajectories may exist in early childhood also remains unclear. In the current study we planned to determine more precisely early life WAZchanges by measuring children at frequent intervals over the first four years.

While genes are known to be robust predictors, evidence demonstrates that lifestyle behaviors established in early childhood life are the main modifiable factors of the weight status $^{[8]}$. Lifestyles, such as eating behavior, physical activity, media exposure and sleeping, are likely to be key factors influencing the risk of childhood overweight/obesity. However, both observational and interventional studies have reported inconsistent results ${ }^{[13-20]}$. Furthermore, two reviews proposed that the combination of obesity-associated risk factors (e.g. low physical activity, more sedentary time, and poor sleep) may have an effect in a way that differs from their impacts when studied in isolation ${ }^{[21,22]}$. Similarly, synergistic effects of rapid WAZ-change and risk lifestyle behaviors (e.g. higher eating appetite, lower physical activity, more media 
exposure and shorter sleep duration) on childhood adiposity in early life have not been characterized, because they were most often studied independently ${ }^{[12,15-17,23]}$. Therefore, from a prevention perspective, insight into the combined effects of rapid weight gain and early childhood lifestyle factors on risk of childhood overweight/obesity is required.

Therefore, we used a birth cohort with nine follow-up time points (at birth, 3, 6, 9, 12, 18, 24, 36, and 48 months) to characterize WAZ-change trajectories, to determine the periods with the greatest WAZ-change, and to investigate independent and combined effects of WAZ-change trajectories with early lifestyle factors on children's adiposity indicators at age of 4 years old. Based on our prior clinical observations, we hypothesized that WAZ-change trajectories in early life could be categorized into distinct patters, such as steady WAZ-change or early infancy rapid WAZ-change trajectory, and the greatest WAZ-change could be occurred very earlier as compared to later life in the first four years. We also hypothesized that distinct growth patterns not only have an independent influence, but also an interactive effect with childhood lifestyle factors on obesity status. For example, individuals with early infancy rapid growth trajectory combined with unhealthy behaviors (i.e. higher appetite, lower physical activity, more media exposure and shorter sleep duration) may have a much higher risk of obesity conditions in later life when compared with those with steady growth pattern and without lifestyle risk factors.

\section{Methods}

\subsection{Participants}

The Shanghai Sleep Birth Cohort Study (SSBCS), an ongoing mother-child birth cohort recruited from the general population, aims to investigate the effects of perinatal and early life environmental and behavioral factors on child growth and development. Detailed information has been described previously ${ }^{[24]}$. Briefly, the recruitment was conducted at the Obstetric Clinic of Renji Hospital (Shanghai, China), which is adjacent to our unit, from May 2012 to July 2013. There were three screening stages based on the predefined inclusion and exclusion criteria (Figure 1). For the screening steps, all the pregnant women who met the inclusion criteria were approached by our research team and 431 candidates (i.e. women in late pregnancy carrying a single baby) were identified. Finally, 262 women with full-term newborns agreed to participate and attend the scheduled follow-up visits in our study. In the first follow-up stage $(n=262)$, we carried out a person-to-person interview at 9 time points, namely late pregnancy, birth within 3 days, 42 days' postpartum ( \pm 3 days), and 3, 6, 9, 12, 18, and 24 months after birth ( \pm 7 days). Due to losing interest for further follow-up, moving away, busy work schedule and other reasons, 25 participants dropped out in this stage. In the second follow-up stage $(n=237)$, person-to- 
person interviews at age of 36, 48 and 72 months ( \pm 1 month) were conducted. The 6-year follow-up has not yet completed; therefore, the current study used the 4-year data.

The birth cohort protocol was approved by the Shanghai Children's Medical Center Human Ethics Committee (SCMCIRB-2012033). All participants provided written informed consent, which was renewed before commencing each stage of data collection. All families received $¥ 50$ in remuneration at each follow-up visit.

\subsection{Measures}

2.2.1 Anthropometric measurement-All measurements were undertaken according to standardized protocols by trained research staff. Child weight and length/height were obtained with light clothes and without shoes at each visit. Weight was measured using calibrated scales (0-2 years, Seca 335) and electronic personal scales (3-4 years, Seca 877). Recumbent length (0-2 years) was measured on the same calibrated scale as used for weight and standing height (2-4 years) was measured using a stadiometer (Seca 206). Weight-for-age z-score (WAZ) was determined using the least mean square method according to the World Health Organization growth reference ${ }^{[25]}$. At the age of 4 years old, the following measurements without clothes were taken: child waist circumference using the Ergonomic circumference measuring tape (Seca 201), biceps circumference using the Heochstmass measuring tape, triceps and subscapular skinfold thicknesses using the Harpenden skinfold caliper. We calculated BMI value from weight being divided by the square of height, computed waist-to-height ratio (WHtR) from waist circumference divided by height and summed the triceps and subscapular skinfold thicknesses as a measure of subcutaneous fat for children at age 4 years.

2.2.2 Eating behaviors-At the age of 2 and 4 years old, parents reported child eating behaviors using the Children's Eating Behavior Questionnaire (CEBQ) ${ }^{[26]}$, which has been validated in different populations including Chinese ${ }^{[27]}$. The original CEBQ is a 35 -item measure constructed in UK children designed to assess 8 dimensions ${ }^{[26]}$. Considering its applicability (sensitive to cultural disparities) to Chinese population ${ }^{[28]}$, perhaps difficulty in identifying negative moods in early childhood and the lack of correspondence between questionnaire and laboratory food intake measures for emotional eating ${ }^{[29]}$, we only used the following four dimensions in the present study: food responsiveness (FR), to assess responsiveness to external food cues; enjoyment of food (EF), to investigate the child's general appetite; satiety responsiveness (SR), to evaluate the sensitivity to internal satiety cues; and food fussiness (FF), to assess a common eating behavior with limited variety or quantity of foods. The four subscales that we selected had good test-retest reliability (Coefficients $(r)=0.83$, 
0.87, 0.85 and 0.87 respectively) and high internal consistency (Cronbach's $\alpha=0.82,0.91,0.83$ and 0.91 respectively) ${ }^{[26]}$. A higher score for FR and EF, as well as a lower score for SR and FF equate to a greater appetite rating.

2.2.3 Physical activity time - At the age of 3 years old, child's outdoor playtime, as a proxy for physical activity, was assessed using a validated survey question ${ }^{[30]}$. That is, parents reported how much time their child spent on playing outdoors per day on a typical weekday and on a typical weekend in the past month. A weighted outdoor playtime per day was calculated by ((hours/weekday*5 + hours/weekend*2) / 7).

2.2.4 Media exposure time-At the age of 2 and 4 years old, parents reported the child's media exposure time including watching TV or DVDs, using a computer or IPAD, as well as playing with electronic games on a typical weekday and on a typical weekend day in the last month. Total media exposure time was summed and a weighted average was calculated using the same function as physical activity time at each age. We also categorized media exposure time into $<1$ and $\geq 1$ hour/day based on the media recommendations of the American Academy of Pediatrics (AAP) for children who aged 2-5 years old.

2.2.5 Total sleep duration-At the age of 2 years old, child's total sleep duration was summed from daytime and nighttime sleep duration using the Brief Infant Sleep Questionnaire $(\mathrm{BISQ})^{[31]}$. At the age of 4 years old, the Children's ChronoType Questionnaire (CCTQ ${ }^{[32]}$ was utilized. Parents were asked about their child's bedtime and wake-up time as well as their daytime sleep on a typical weekday and on a typical weekend day in the last month. Both nighttime sleep on weekdays and weekends were calculated, then a weighted total sleep time was calculated.

2.2.6 Other variables - Demographic data reported by mothers were collected in late pregnancy and at birth. Maternal pre-pregnancy BMI, total gestational weight gain between prepregnancy and the last clinically recorded weight within two weeks after delivery, and paternal BMI values were obtained from the Obstetric Clinic. We also assessed maternal sleep quality, depressive symptom and anxiety status at the first visit using the Pittsburgh Sleep Quality Index $^{[33]}$, the Center for Epidemiological Survey-Depression Scale ${ }^{[34]}$ and the State-Trait Anxiety Index ${ }^{[35]}$, respectively, all of which are standardized questionnaires and are widely used in China. Details can be found in our previous articles ${ }^{[24,36]}$. On each postnatal follow-up (until 24 months' postpartum), the mothers were asked to report the feeding method (exclusive breastfeeding, mixed feeding, or bottle feeding). Infant feeding method during the first 3 months was categorized as exclusive breastfeeding and non-exclusive breastfeeding (mixed feeding and 
bottle feeding). Breastfeeding duration (weaning time) was categorized as $<6$ months, 6 to 12 months or $\geq 12$ months. Infant dietary intake was assessed at 6 months using a 3 -day food diary, which asked parents/caregivers to list all foods and drinks (including water) that the child consumed and to record the time, content and quantity of each meal. Electronic kitchen scales (CAMRY- EK3550-31P, Beijing, China) and precise instructions were provided to assist parents in quantifying their child's food intake. Total energy, protein, fat, and carbohydrate intake were estimated by research staff using the China Food Composition Tables.

\subsection{Statistical analysis}

Maternal and infant characteristics were presented as mean (95\% confidence interval, CI) or frequency (percentage), and differences in characteristics between lost and retained children when aged 4 years old were assessed by the t-test and $\chi^{2}$ test, for continuous variables and categorical variables, respectively. To address our aims, the statistical analyses were performed in the following steps.

In step 1, to identify the WAZ-change trajectories, the WAZ-changes were calculated and group-based trajectory modeling (GBTM) was used ${ }^{[37]}$. We quantified WAZ-changes during each age interval as the subtraction between adjacent measurement points with positive differences representing WAZ gain and negative differences representing WAZ loss, and defined WAZ-change $\geq+0.67$ over two time points as rapid change ${ }^{[10]}$. The following eight time intervals, i.e. $0-3,3-6,6-9,9-12,12-18,18-24,24-36$, and 36-48 months were used to characterize the WAZ-change trajectories. On average, each child had 4.9 (95\% CI: 4.7, 5.2) WAZ-change observations, and only children with a minimum of three observations were included. The GBTM assesses the average pattern of WAZ-change over time and assumes that individuals belong to the same underlying population represented by a single growth curve. The best-fitting model was chosen based on the largest Bayesian information criterion and each trajectory included at least $5 \%$ of the sample size.

In step 2, we tested the independent and combined effects of WAZ-change trajectories and different lifestyles on each adiposity outcome using a multivariate linear regression model, controlling for multiple baseline factors (family income, gestational age of the child at delivery, maternal pre-pregnancy BMI, paternal BMI, newborn weight over the first three days, sex and energy intake at six months) Firstly, we assessed the effects of WAZ-change trajectories and each lifestyle factor (i.e. FR, EF, SR, FF, outdoor playtime, media exposure time and total sleep duration) on adiposity measures solitarily. Secondly, both WAZ-change trajectories and lifestyle factors that had a significant effect on adiposity measures, were imported into the same model 
to test their independent effects on adiposity indicators. Thirdly, to aid clinical interpretation, lifestyle factors that had an independent effect on adiposity measures were then categorized as dichotomous variables by median levels, except for media exposure time whose cut-off value was defined as one hour ${ }^{[13]}$. As, only four eating behaviors had significant effects on adiposity measures, they were dichotomized and put into the next combined analyses. Fourthly, we combined the WAZ-change trajectories and each of these four dichotomous eating behaviors to examine a range of joint effects on the adiposity outcomes ${ }^{[38]}$. Children with lower risk factors of overweight/obesity were used as the reference group in the analyses, e.g. steady growth pattern and lower FR score, or steady growth pattern and higher SR score. Post-hoc pairwise comparisons were performed with Bonferroni multiple comparison test. Notably, the lifestyle factors investigated at the age of 2 and 4 years old were averaged as the childhood exposure during this period and to reduce the number of variables in further multivariate regression models, as had been done in a previous study ${ }^{[39]}$.

In step 3, to avoid loss of statistical power due to missing data, we performed a multiple imputation using chained equations (MICE) with 100 imputed data sets and 1,000 burn-ins for each dataset to estimate the missing values ${ }^{[0]}$. To test whether substantial differences existed due to imputation, we compared the results before and after the data imputation.

All analyses were performed by using the Stata SE 15.0 software (Stata Corp, College Station, TX, USA), and the statistical significance was set at a 2-sided P value less than 0.05 .

\section{Results}

\subsection{Descriptive information}

Overall, 262 mother-child pairs participated, with 221 (84.4\%) cases having at least three WAZ-change data points to characterize trajectories, and 209 (79.8\%) cases finishing the 4year measurement to evaluate childhood adiposity outcomes (Figure 1). No significant differences due to attrition were found at the age of 4 years (Table 1). Of the 262 mother-infant pairs with complete data at birth, 50.4\% were boys. The majority of women had a college or higher level of education (91.2\%). Maternal age at delivery was 29.5 (95\% CI: 29.1, 29.9) years old, and child's birth weight was 3.31 (95\% CI: 3.26, 3.36) kg.

\subsection{WAZ-change trajectories}

Mean WAZ-change decreased from 0.80 (95\% CI: 0.67, 0.93) over 0-3 months and 0.26 (95\% CI: $0.19,0.34)$ over 3-6 months to just below the zero baseline in subsequent age intervals. The percentage of cases experiencing rapid weight change dropped with a similar trend, from 
$54.0 \%$ over $0-3$ months and $22.9 \%$ over $3-6$ months to less than $8 \%$ in subsequent age intervals (Table S1).

Over the first four years of life, two distinct trajectories that best represent the dynamic WAZ-changes were detected using GBTM (Figure 2). Given the recommended cut-off value of the rapid weight change (WAZ-change $>+0.67$ ), two groups were identified: WAZ-changes near to zero at all age intervals (group $1, \mathrm{~N}=84,38.0 \%$ ); and a rapid WAZ-change over 0-3 months, while fluctuating near zero afterwards (group 2, $\mathrm{N}=137 ; 62.0 \%$ ). Therefore, we defined group 1 as "steady" and group 2 as "early infancy" rapid WAZ-change trajectory.

Further analyses also indicated a significant difference in the absolute WAZ-change and weight increment $(\mathrm{kg})$ between the two trajectories (Table S1). The first 6 months, in particular the first 3 months with the largest WAZ-change, was the critical period, and children with "early infancy" rapid WAZ-change trajectory showed a non-stable weight pattern.

\subsection{Independent effects}

Most of the mother-child characteristics were similar between steady and early infancy rapid groups (Table S2). However, children in the early infancy rapid group were more likely to have shorter gestational age, shorter birth length and lower birth weight. The early infancy rapid group was also more likely to have higher EF and lower FF scores, in addition to having less outdoor playtime in early childhood, even after adjusting for confounders.

After adjusting for mother-child characteristics, the early infancy rapid group had significantly higher adiposity status (except for WHtR) compared with the steady group, with the regression coefficient of 0.90 (95\% CI: 0.37, 1.44) kg for weight, 0.93 (95\% CI: $0.49,1.37$ ) $\mathrm{kg} / \mathrm{m}^{2}$ for BMI, 1.90 (95\% CI: 0.68, 3.12) cm for waist circumference, 1.05 (95\% CI: 0.62, 1.48) $\mathrm{cm}$ for biceps circumference, and 2.57 (95\% CI: 1.13, 4.01) mm for subcutaneous fat (Model 1a in Table 2). Meanwhile, scores of FR and EF had positive effects, and, SR and FF had negative effects on adiposity measures with the exception of WHtR (Model 1b-1e in Table 2). Further analyses showed that either the WAZ-change trajectory or four eating subscale scores independently had significant associations on adiposity measures except for WHtR (Model 2a2d in Table 2). However, the other lifestyle factors, such as outdoor playtime, media exposure time and total sleep duration had no significant association with adiposity measures (Model 1f$1 \mathrm{~h}$ in Table 2).

\subsection{Combined effects}

Combined effects were shown in Table 3 and Figure 3 (post-hoc multiple comparison test). 
While we did not find statistically interactive effect of growth trajectory and each eating behavior (Table S3), we did find significantly combined effects of the WAZ-change trajectory with three in four subscales of eating behaviors alone on the majority of childhood adiposity outcomes (except for WHtR) at the age of 4 years, all of which were in the absence of independent effects from early infancy rapid trajectory and eating behaviors. Specifically, compared to children with steady WAZ-change trajectory and lower appetite score (the reference group), children with early infancy rapid trajectory and larger appetite rating (i.e. higher FR score, lower SR and FF score) had significantly more waist circumference and subcutaneous fat, children with early infancy rapid trajectory and higher FR or lower FF score had obliviously more weight, additionally, children with early infancy rapid trajectory and higher FR score had apparently more BMI and subcutaneous fat.

\subsection{Sensitivity analysis}

Our sensitivity analysis found that the results of multiple-imputation had limited effect on the results of complete-case analysis with the exception of the results for the SR score (Table S4-S6 and Figure S1).

\section{Discussion}

To the best of our knowledge, no studies have characterized different trajectories of WAZchange among full-term children and determined their combined effects with lifestyle factors on adiposity outcomes in early childhood life. There are several important findings. We found (1) a high percentage of infants with rapid growth in our cohort, (2) characterized two distinct WAZ-change trajectories from birth to 4 years - one was a steady trajectory and the other an early infancy rapid WAZ-change trajectory - with the critical window for this change occurring over 0-6 months, especially with the greatest change over 0-3 months, and (3) identified significant independent and combined effects of WAZ-change trajectories and eating behaviors on childhood adiposity markers at age 4 years, indicating that both early growth and appetite have impacts that manifest in later childhood.

According to the widely used cut-off for a rapid WAZ-change $(>+0.67)$ between any two measurement points, $54 \%$ of infants during the first three months and $64 \%$ during the first six months showed rapid growth in our study, which was a much higher percentage than that reported from other countries, e.g. $19 \%$ in Japan ${ }^{[41]}$ and $30 \%$ in the $\mathrm{USA}^{[42]}$ over $0-4$ months, and $22 \%$ in Australia ${ }^{[43]}, 24 \%$ in Spain $^{[44]}, 28 \%$ in Northeast Brazil ${ }^{[45]}$ over 0-6 months. The present study characterized two WAZ-change trajectories from birth to 4 years: only $38 \%$ of children had a steady WAZ-change growth pattern, and the remainder of the children exhibiting 
an early infancy rapid growth with the weight increment in $0-3$ months interval at the $85^{\text {th }}$ percentile (boys) or the $95^{\text {th }}$ percentile (girls) according to the WHO standard ${ }^{[46]}$. While a limited number of studies have characterized WAZ-change trajectories in the same way as the present study, several studies have identified different absolute weight or BMI trajectories ${ }^{[47]}$. Most reported a combination of stable (low, medium or high) and ascending (early or later) trajectories over time, which might be somewhat clinically similar to the steady and early infancy rapid growth trajectories in the current research. However, the percentages with an early ascending trajectory in other studies were lower than that presented in this study.

Children with early infancy rapid growth in our cohort were more likely to have a lower birth weight, which is in line with findings of a previous study ${ }^{[8]}$, and is likely to be related in part to a significantly shorter pregnancy (albeit only a few days). However, all participants in the current study were normal pregnant women with healthy full-term neonates, $97.6 \%$ of children were appropriate for gestational age (post hoc analysis), and no significant difference in the method of delivery was found between the two growth trajectories. Future larger cohort studies should be conducted to confirm our findings. As for the postnatal factors, the rapid WAZ-change pattern may be attributable to the disparities in parents' feeding beliefs, attitudes, knowledge or practices related to cultural perceptions. Chinese families are more likely to consider a baby with more weight to be healthy. Consequently, over-nutrition combined with early consumption of formula, water-based drinks, fruit juice and introduction of complementary foods will be much more likely to occur ${ }^{[48,49]}$.

Several studies have explored rapid infant weight change, but failed to demonstrate its "exact critical age window". That is, they either investigated only one age interval ${ }^{[10,11]}$ or reported conflicting evidence regarding the specific critical period. Some reported early infancy ${ }^{[50]}$, some reported later infancy ${ }^{[51]}$ or later life ${ }^{[52]}$, while others indicated both ${ }^{[53]}$. These discrepancies could be due to differences in length of follow-up, age intervals, or ethnicity. Characterizing distinct WAZ-change trajectories not only can precisely illustrate the dynamic weight changes in size over time, but also can determine the critical rapid growth window over the whole growth picture ${ }^{[47]}$. In our four-year birth cohort study with nine follow-up visits, we identified two different WAZ-change trajectories, which suggested that the first six months, particularly the first three months, was the most critical growth period. One study showed that infants with rapid weight change over the first one month tended to continue to exhibit rapid growth in the following months ${ }^{[54]}$. The two distinct growth patterns from our data are particularly useful for clinicians and health professionals to design early-life interventions by targeting infants with rapid weight gain in the first few months. 
The current study suggested that it is the eating behavior, not the other lifestyle factors tested (i.e. outdoor playtime, media exposure time and total sleep duration) in early childhood, that significantly predicted later adiposity status. Previous research has found that a larger appetite rating (e.g. greater FR and EF scores; lower SR and FF scores) was associated with adiposity in children ${ }^{[55,56]}$. The underlying mechanisms may be related to the higher FR, leading to more frequent eating when exposed to food that triggers brain-reward pathways; and to the lower SR, leading to more food being eaten on each eating occasion again related to a neuroendocrine feedback loop ${ }^{[57]}$. One prior study also observed that a higher FR score was correlated to a greater energy intake. In addition a higher EF score was associated with a greater energy intake and more food eaten without hunger, while children with a higher SR score had a lower energy intake, a smaller food intake without hunger and a better average caloric compensation ${ }^{[58]}$.

In the present study, we observed a significantly joined effect between growth trajectory and each eating behavior on most of adiposity indicators. Our study extends the current literature, in which WAZ-change trajectories not only have an independent effect, but also a biologically combined impact with children's eating behaviors (such as FR, SR and FF) on childhood adiposity measures, which suggested a synergistic effect between them in early childhood. Thus, children who had an early infancy rapid growth with a larger appetite score were more likely to develop a higher adiposity status compared with children showing steady growth with a lower appetite score. Some studies have indicated that higher weight gain and weight status in early life can positively predict subsequent energy intake, FR and EF, as well as negatively predicting later $\mathrm{SR}^{[59,60]}$. Previous studies also have demonstrated that a larger appetite would facilitate early rapid weight gain ${ }^{[61,62]}$. The interactive influence between them was more likely to exacerbate the occurrence and development of childhood and adulthood overweight/obesity. If not treated proactively, they can persist and are not easy to improve by themselves. Therefore, control of rapid growth in the first three to six months of life, followed by strategies to reduce the potential modifying effects of eating behaviors in preschool children $^{[63]}$, in a period when appetite traits are becoming established as many children become autonomous eaters and experience greater socialization of eating ${ }^{[64,65]}$, are required.

While eating behaviors have a genetic background ${ }^{[66]}$, they are also influenced by environmental factors. Studies have suggested that the critical window for altering eating behaviors might occur after birth ${ }^{[62]}$, and parental feeding practices are potentially a good behavior to target early in life ${ }^{[67,68]}$. Sustaining exclusive breastfeeding and delaying complementary feeding until 6 months can not only promote maternal feeding styles with less controlling and more responsive to infant cues of hunger and satiety, but also can modify infants' 
self-regulation of energy intake and $\mathrm{SR}^{[61,69]}$. While early children characterized of rejection of new foods and increased autonomy and self-regulation abilities, parents can also play a critical role in shaping their children's eating behaviors by avoiding maladaptive feeding strategies such as pressure to eat, using food as a reward and unhealthy food habits ${ }^{[64,65,68]}$.

Our findings should be considered in the context of potential limitations. Firstly, our samples were from Shanghai, a relatively socioeconomically advantaged city in China. The majority of parents were university educated and had family incomes at or above the national average. Hence, the findings might not be generalizable to the national population. However, Shanghai is representative of most of the cities that have evolved from developing to developed status. Therefore, our findings are likely to reflect what will happen in other developing cities and can assist clinicians designing early-life interventions in this socioeconomic setting. Samples with a wider range of socioeconomic status should be collected in the future work. Secondly, our small sample size and the attrition over time may reduce the power to detect some associations. However, no significant differences in maternal or child characteristics at the age of 4 years were found in those who stayed in the cohort versus those that did not; additionally, we did not find attrition bias based on the results of sensitivity analyses (nearly identical results between multiple-imputation and complete-case analyses) suggesting a relatively robust result. Thirdly, parent-reported behavioral data may be subject to recall bias. Objective measurements should be collected in the future studies. Fourthly, we did not obtain children's caloric intake in other periods except for 6 months. The caloric intake in other periods should be also assessed in the future research to identify differences between the two WAZ-change growth patterns. Fifthly, the multiple comparisons performed in our study could increase the type I errors. Finally, given its observational nature, we cannot claim the causality and cannot exclude the possibility that our results may be influenced by residual and unmeasured or unknown confounding factors.

\section{Conclusions}

Our study has identified two distinct weight change trajectories, highlighted the first six months, particularly the first three months, as a possible critical growth window, and showed a significant effect of weight change trajectory and eating behaviors (e.g. FR, SR and FF), either alone or in combination, on the majority of adiposity measures at the age of 4 years. These findings extend the current literature and provide a potentially valuable model to aid clinicians and health professionals in designing early-life interventions targeting specific populations, specific ages and specific lifestyle behaviors to prevent childhood overweight/obesity. Our cohort is ongoing, and future follow-up on these children will be essential to indicate whether our observations may have long-term repercussions. Further studies with larger sample size, 
longer timeframes, and more exact evaluation of lifestyle behaviors in different populations are needed to verify our findings.

\section{Acknowledgments}

We are grateful to thank Dr. Shilu Tong for his comments in the early drafts of this manuscript, and Hangjun Gong for providing valuable assistance with statistical analyses. We also thank the families who participated in our cohort, and were followed up at each scheduled visit.

\section{Authors' Contributions}

Ms. Lin designed the study, analyzed and interpreted the data, drafted the initial manuscript as well as all subsequent drafts, and reviewed and revised the manuscript. Ms. Jiang and Mr. Wang designed the study, and critically reviewed and revised the manuscript. Ms. Sun, Dong, Deng, Meng and Zhu designed the study, managed participant recruitment and data collection, and critically reviewed the manuscript. Dr. Mei, Zhou, Zhang, and Clayton conceptualized and designed the study, provided guidance on the statistical analyses, and critically reviewed the manuscript. Dr. Spruyt designed the study, provided guidance on the statistical analyses, interpreted the data, and critically reviewed and revised the manuscript. Dr. Jiang conceptualized and designed the study, provided guidance on the statistical analyses, interpreted the data, and critically reviewed and revised the manuscript. All the authors read and approved the final manuscript.

\section{Study funding}

The study was supported by Chinese National Natural Science Foundation (81422040, 81773443, 81602868, 81728017, and 11771146), Ministry of Science and Technology (2016YFC1305203), National Health Commission of the People's Republic of China (201002006), Science and Technology Commission Shanghai Municipality (18695840200, 17XD1402800, 2018SHZDZX05, 18JC1420305, and 14441904004), Shanghai Municipal Health Commission (2016ZB0104, 2017ZZ02026, and 20164Y0095), and Shanghai Jiao Tong University (YG2016ZD04). 


\section{Reference}

1. Collaboration NCDRF. Worldwide trends in body-mass index, underweight, overweight, and obesity from 1975 to 2016: a pooled analysis of 2416 population-based measurement studies in 128.9 million children, adolescents, and adults. Lancet. 2017;390(10113):2627-42.

2. Geserick M, Vogel M, Gausche R, Lipek T, Spielau U, Keller E, et al. Acceleration of BMI in Early Childhood and Risk of Sustained Obesity. N Engl J Med. 2018;379(14):1303-12.

3. Afshin A, Forouzanfar MH, Reitsma MB, Sur P, Estep K, Lee A, et al. Health Effects of Overweight and Obesity in 195 Countries over 25 Years. N Engl J Med. 2017;377(1):13-27.

4. Mamrot $\mathrm{P}$, Hanć $\mathrm{T}$. The association of the executive functions with overweight and obesity indicators in children and adolescents: A literature review. Neurosci Biobehav Rev. 2019;107:59-68.

5. Zimmermann E, Bjerregaard LG, Gamborg M, Vaag AA, Sorensen TIA, Baker JL. Childhood body mass index and development of type 2 diabetes throughout adult life-A large-scale danish cohort study. Obesity (Silver Spring). 2017;25(5):965-71.

6. Sonntag D. Why Early Prevention of Childhood Obesity Is More Than a Medical Concern: A Health Economic Approach. Ann Nutr Metab. 2017;70(3):175-8.

7. Nogrady B. Childhood obesity: A growing concern. Nature. 2017;551(7681).

8. Larque E, Labayen I, Flodmark CE, Lissau I, Czernin S, Moreno LA, et al. From conception to infancy - early risk factors for childhood obesity. Nat Rev Endocrinol. 2019;15(8):456-78.

9. Matthews EK, Wei J, Cunningham SA. Relationship between prenatal growth, postnatal growth and childhood obesity: a review. Eur J Clin Nutr. 2017;71(8):919-30.

10. Zheng M, Lamb KE, Grimes C, Laws R, Bolton K, Ong KK, et al. Rapid weight gain during infancy and subsequent adiposity: a systematic review and meta-analysis of evidence. Obes Rev. 2018;19(3):321-32.

11. Iguacel I, Escartin L, Fernandez-Alvira JM, Iglesia I, Labayen I, Moreno LA, et al. Early life risk factors and their cumulative effects as predictors of overweight in Spanish children. Int J Public Health. 2018;63(4):501-12.

12. Barraclough JY, Garden FL, Toelle BG, Marks GB, Baur LA, Ayer JG, et al. Weight Gain Trajectories from Birth to Adolescence and Cardiometabolic Status in Adolescence. J Pediatr. 2019;208:89-95.e4.

13. Bawaked RA. Impact of lifestyle behaviors in early childhood on obesity and cardiometabolic risk in children: Results from the Spanish INMA birth cohort study. Pediatr Obes. 2019:e12590.

14. Jackson SL, Cunningham SA. The stability of children's weight status over time, and the role of television, physical activity, and diet. Prev Med. 2017;100:229-34.

15. Spahić R, Pranjić N. Children's Eating Behaviour Questionnaire: association with BMI in children aged 3-10 years from Bosnia and Herzegovina. Public Health Nutr. 2019;22(18):3360-7.

16. Collings PJ, Kelly B, West J, Wright J. Associations of TV Viewing Duration, Meals and Snacks Eaten When Watching TV, and a TV in the Bedroom with Child Adiposity. Obesity (Silver Spring). 2018;26(10):1619-28.

17. Alderete TL, Ryoo JH, Goran MI, Zhang Z. The cross-sectional and prospective associations between sleep characteristics and adiposity in toddlers: Results from the GET UP! Study. Pediatr Obes. 2019;14(11):e12557.

18. Taylor RW, Gray AR, Heath AM, Galland BC, Lawrence J, Sayers R, et al. Sleep, nutrition, and physical activity interventions to prevent obesity in infancy: follow-up of the Prevention of Overweight in Infancy (POI) randomized controlled trial at ages 3.5 and 5 y. Am J Clin Nutr. 2018;108(2):228-36.

19. Plimier CC, Hewawitharana SC, Webb KL, Au LE, Neumark-Sztainer D, Ritchie LD. Community-level obesity prevention is not associated with dieting behaviours and weight dissatisfaction in children: The Healthy Communities Study. Pediatr Obes. 2020;15(4):e12594. 
20. Poeta M, Lamberti R, Di Salvio D, Massa G, Torsiello N, Pierri L, et al. Waist Circumference and Healthy Lifestyle Preferences/Knowledge Monitoring in a Preschool Obesity Prevention Program. Nutrients. 2019;11(9).

21. Chaput JP, Saunders TJ, Carson V. Interactions between sleep, movement and other non-movement behaviours in the pathogenesis of childhood obesity. Obes Rev. 2017;18 Suppl 1:7-14.

22. Dulloo AG, Miles-Chan JL, Montani JP. Nutrition, movement and sleep behaviours: their interactions in pathways to obesity and cardiometabolic diseases. Obes Rev. 2017;18 Suppl 1:3-6.

23. Koning M, Hoekstra T, de Jong E, Visscher TL, Seidell JC, Renders CM. Identifying developmental trajectories of body mass index in childhood using latent class growth (mixture) modelling: associations with dietary, sedentary and physical activity behaviors: a longitudinal study. BMC Public Health. 2016;16(1):1128.

24. Lin J, Sun W, Song Y, Dong S, Lin Q, Deng Y, et al. Cohort Profile: The Shanghai Sleep Birth Cohort Study. Paediatr Perinat Epidemiol. 2020 Dec 18. doi: 10.1111/ppe.12738. Epub ahead of print.

25. Group WMGRS. WHO Child Growth Standards: Length/height-for-age, weight-for-age, weight-forlength, weight-for-height and body mass index-for-age: Methods and development. Geneva: World Health Organization. 2006:312.

26. Wardle J, Guthrie CA, Sanderson S, Rapoport L. Development of the Children's Eating Behaviour Questionnaire. J Child Psychol Psychiatry. 2001;42(7):963-70.

27. Mallan KM, Liu WH, Mehta RJ, Daniels LA, Magarey A, Battistutta D. Maternal report of young children's eating styles. Validation of the Children's Eating Behaviour Questionnaire in three ethnically diverse Australian samples. Appetite. 2013;64:48-55.

28. Jiang X, Yang X, Zhang Y, Wang B, Sun L, Shang L. Development and preliminary validation of Chinese preschoolers' eating behavior questionnaire. PLoS One. 2014;9(2):e88255.

29. Blissett J, Farrow C, Haycraft E. Relationships between observations and parental reports of 3-5 year old children's emotional eating using the Children's Eating Behaviour Questionnaire. Appetite. 2019; $141: 104323$.

30. Burdette HL, Whitaker RC, Daniels SR. Parental report of outdoor playtime as a measure of physical activity in preschool-aged children. Arch Pediatr Adolesc Med. 2004;158(4):353-7.

31. Sadeh A, Mindell JA, Luedtke K, Wiegand B. Sleep and sleep ecology in the first 3 years: a web-based study. J Sleep Res. 2009;18(1):60-73.

32. Werner H, Lebourgeois MK, Geiger A, Jenni OG. Assessment of chronotype in four- to eleven-year-old children: reliability and validity of the Children's Chronotype Questionnaire (CCTQ). Chronobiol Int. 2009;26(5):992-1014.

33. Tsai PS, Wang SY, Wang MY, Su CT, Yang TT, Huang CJ, et al. Psychometric evaluation of the Chinese version of the Pittsburgh Sleep Quality Index (CPSQI) in primary insomnia and control subjects. Qual Life Res. 2005;14(8):1943-52.

34. Cheung CK, Bagley C. Validating an American scale in Hong Kong: the Center for Epidemiological Studies Depression Scale (CES-D). J Psychol. 1998;132(2):169-86.

35. Shek DT. The Chinese version of the State-Trait Anxiety Inventory: its relationship to different measures of psychological well-being. J Clin Psychol. 1993;49(3):349-58.

36. Wang G, Deng Y, Jiang Y, Lin Q, Dong S, Song Y, et al. Trajectories of sleep quality from late pregnancy to 36 months postpartum and association with maternal mood disturbances: a longitudinal and prospective cohort study. Sleep. 2018;41(12).

37. Nagin DS. Group-Based Modeling of Development. Harvard University Press. 2005.

38. Andersson T, Alfredsson L, Kallberg H, Zdravkovic S, Ahlbom A. Calculating measures of biological interaction. Eur J Epidemiol. 2005;20(7):575-9. 
39. Boeke CE, Storfer-Isser A, Redline S, Taveras EM. Childhood sleep duration and quality in relation to leptin concentration in two cohort studies. Sleep. 2014;37(3):613-20.

40. Sterne JA, White IR, Carlin JB, Spratt M, Royston P, Kenward MG, et al. Multiple imputation for missing data in epidemiological and clinical research: potential and pitfalls. Bmj. 2009;338:b2393.

41. Mine T, Tanaka T, Nakasone T, Itokazu T, Yamagata Z, Nishiwaki Y. Maternal smoking during pregnancy and rapid weight gain from birth to early infancy. J Epidemiol. 2017;27(3):112-6.

42. Wang G, Johnson S, Gong Y, Polk S, Divall S, Radovick S, et al. Weight Gain in Infancy and Overweight or Obesity in Childhood across the Gestational Spectrum: a Prospective Birth Cohort Study. Sci Rep. 2016;6:29867.

43. Sutharsan R, O'Callaghan MJ, Williams G, Najman JM, Mamun AA. Rapid growth in early childhood associated with young adult overweight and obesity--evidence from a community based cohort study. $\mathrm{J}$ Health Popul Nutr. 2015;33:13.

44. Valvi D, Mendez MA, Garcia-Esteban R, Ballester F, Ibarluzea J, Goni F, et al. Prenatal exposure to persistent organic pollutants and rapid weight gain and overweight in infancy. Obesity (Silver Spring). 2014;22(2):488-96.

45. Goncalves FC, Amorim RJ, Eickmann SH, Lira PI, Lima MC. The influence of low birth weight body proportionality and postnatal weight gain on anthropometric measures of 8-year-old children: a cohort study in Northeast Brazil. Eur J Clin Nutr. 2014;68(8):876-81.

46. Group WMGRS. WHO Child Growth Standards: Growth velocity based on weight, length and head circumference: Methods and development. Geneva: World Health Organization. 2009:22-5.

47. Mattsson M, Maher GM, Boland F, Fitzgerald AP, Murray DM, Biesma R. Group-based trajectory modelling for BMI trajectories in childhood: A systematic review. Obes Rev. 2019;20(7):998-1015.

48. Lindsay AC, Le Q, Greaney ML. Infant Feeding Beliefs, Attitudes, Knowledge and Practices of Chinese Immigrant Mothers: An Integrative Review of the Literature. Int J Environ Res Public Health. $2017 ; 15(1)$.

49. Bolton KA, Kremer P, Hesketh KD, Laws R, Kuswara K, Campbell KJ. Differences in infant feeding practices between Chinese-born and Australian-born mothers living in Australia: a cross-sectional study. BMC Pediatr. 2018;18(1):209.

50. Min J, Li J, Li Z, Wang Y. Impacts of infancy rapid weight gain on 5-year childhood overweight development vary by age and sex in China. Pediatr Obes. 2012;7(5):365-73.

51. Karp RJ, Winkfield-Royster T, Weedon J. Relation of growth rate from birth to three months and four to six months to body mass index at ages four to six years. J Nutr Metab. 2012;2012:158643.

52. Kramer MS, Zhang X, Martin RM, Oken E, Aris IM, Yang S. Growth During Infancy and Early Childhood and Its Association with Metabolic Risk Biomarkers at 11.5 Years. Am J Epidemiol. 2019;pii: kwz234.

53. Braun JM, Kalkwarf HJ, Papandonatos GD, Chen A, Lanphear BP. Patterns of early life body mass index and childhood overweight and obesity status at eight years of age. BMC Pediatr. 2018;18(1):161.

54. Cole TJ, Singhal A, Fewtrell MS, Wells JC. Weight centile crossing in infancy: correlations between successive months show evidence of growth feedback and an infant-child growth transition. Am J Clin Nutr. 2016;104(4):1101-9.

55. Quah PL, Chan YH, Aris IM, Pang WW, Toh JY, Tint MT, et al. Prospective associations of appetitive traits at 3 and 12 months of age with body mass index and weight gain in the first 2 years of life. BMC Pediatr. 2015;15:153.

56. Llewellyn CH, Trzaskowski M, van Jaarsveld CHM, Plomin R, Wardle J. Satiety mechanisms in genetic risk of obesity. JAMA Pediatr. 2014;168(4):338-44. 
57. Syrad H, Johnson L, Wardle J, Llewellyn CH. Appetitive traits and food intake patterns in early life. Am J Clin Nutr. 2016;103(1):231-5.

58. Carnell S, Wardle J. Measuring behavioural susceptibility to obesity: validation of the child eating behaviour questionnaire. Appetite. 2007;48(1):104-13.

59. van Deutekom AW, Chinapaw MJ, Vrijkotte TG, Gemke RJ. The association of birth weight and postnatal growth with energy intake and eating behavior at 5 years of age - a birth cohort study. Int $\mathrm{J}$ Behav Nutr Phys Act. 2016;13:15.

60. Derks IPM, Sijbrands EJG, Wake M, Qureshi F, van der Ende J, Hillegers MHJ, et al. Eating behavior and body composition across childhood: a prospective cohort study. Int J Behav Nutr Phys Act. 2018;15(1):96.

61. Buvinger E, Rosenblum K, Miller AL, Kaciroti NA, Lumeng JC. Observed infant food cue responsivity: Associations with maternal report of infant eating behavior, breastfeeding, and infant weight gain. Appetite. 2017;112:219-26.

62. Lagisz M, Blair H, Kenyon P, Uller T, Raubenheimer D, Nakagawa S. Transgenerational effects of caloric restriction on appetite: a meta-analysis. Obes Rev. 2014;15(4):294-309.

63. de Lauzon-Guillain B, Clifton EA, Day FR, Clement K, Brage S, Forouhi NG, et al. Mediation and modification of genetic susceptibility to obesity by eating behaviors. Am J Clin Nutr. 2017;106(4):9961004.

64. Rahill S, Kennedy A, Kearney J. A review of the influence of fathers on children's eating behaviours and dietary intake. Appetite. 2019;147:104540.

65. Eichler J, Schmidt R, Poulain T, Hiemisch A, Kiess W, Hilbert A. Stability, Continuity, and BiDirectional Associations of Parental Feeding Practices and Standardized Child Body Mass Index in Children from 2 to 12 Years of Age. Nutrients. 2019;11(8).

66. Monnereau C, Jansen PW, Tiemeier H, Jaddoe VW, Felix JF. Influence of genetic variants associated with body mass index on eating behavior in childhood. Obesity (Silver Spring). 2017;25(4):765-72.

67. Anzman-Frasca S, Ventura AK, Ehrenberg S, Myers KP. Promoting healthy food preferences from the start: a narrative review of food preference learning from the prenatal period through early childhood. Obes Rev. 2018;19(4):576-604.

68. Scaglioni S, De Cosmi V, Ciappolino V, Parazzini F, Brambilla P, Agostoni C. Factors Influencing Children's Eating Behaviours. Nutrients. 2018;10(6).

69. Taylor RW, Williams SM, Fangupo LJ, Wheeler BJ, Taylor BJ, Daniels L, et al. Effect of a Baby-Led Approach to Complementary Feeding on Infant Growth and Overweight: A Randomized Clinical Trial. JAMA Pediatr. 2017;171(9):838-46. 
Table 1 Maternal-child characteristics between retained and lost children at four years (non-imputed data).

\begin{tabular}{|c|c|c|c|c|c|}
\hline & $\begin{array}{c}\text { All } \\
(n=262)\end{array}$ & $\begin{array}{c}\text { Retained } \\
(n=209)\end{array}$ & $\begin{array}{c}\text { Lost } \\
(n=53)\end{array}$ & $t / \chi^{2}$ & $\begin{array}{c}P \\
\text { value }\end{array}$ \\
\hline \multicolumn{6}{|l|}{ Maternal (pregnancy) } \\
\hline Highest parents education & & & & 1.22 & 0.544 \\
\hline High school or lower & $23(8.8)$ & $18(8.6)$ & $5(9.4)$ & & \\
\hline College or higher & $239(91.2)$ & $190(91.4)$ & $48(90.6)$ & & \\
\hline Maternal age at birth, year & $29.47(29.08,29.86)$ & $29.70(29.24,30.15)$ & $28.71(27.95,29.47)$ & -2.00 & 0.046 \\
\hline Gestational age, week & $39.65(39.53,39.77)$ & $39.63(39.49,39.77)$ & $39.71(39.47,39.96)$ & -0.10 & 0.922 \\
\hline Pre-pregnancy BMI, kg/m² & $20.61(20.30,20.93)$ & $20.72(20.34,21.10)$ & $20.25(19.72,20.78)$ & -0.89 & 0.377 \\
\hline Paternal BMI, $\mathrm{kg} / \mathrm{m}^{2}$ & $24.37(23.86,24.88)$ & $24.55(23.93,25.17)$ & $23.77(22.95,24.59)$ & -1.58 & 0.116 \\
\hline Gestational weight gain, $\mathrm{kg}$ & $16.28(15.75,16.81)$ & $16.11(15.49,16.73)$ & $16.96(15.97,17.94)$ & -1.26 & 0.208 \\
\hline Maternal sleep quality & $96(40.7)$ & $80(42.1)$ & $16(34.8)$ & 0.82 & 0.364 \\
\hline Maternal state anxiety & $20(8.2)$ & $15(7.7)$ & $5(10.2)$ & 0.32 & 0.574 \\
\hline Maternal trait anxiety & $29(12.0)$ & $22(11.3)$ & $7(14.6)$ & 0.38 & 0.536 \\
\hline Maternal depression & $30(12.0)$ & $22(11.0)$ & $8(16.0)$ & 0.97 & 0.324 \\
\hline \multicolumn{6}{|l|}{ Newborn } \\
\hline Male & $132(50.4)$ & $105(50.2)$ & $27(50.9)$ & 0.01 & 0.927 \\
\hline Vaginal delivery & $100(39.4)$ & $78(38.6)$ & $22(42.3)$ & 0.24 & 0.627 \\
\hline Birth order 1 & $237(92.9)$ & $188(93.1)$ & $49(92.5)$ & 0.02 & 0.537 \\
\hline Birth season & & & & 3.24 & 0.356 \\
\hline Spring, Mar to May & $57(21.8)$ & $44(21.1)$ & $13(24.5)$ & & \\
\hline Summer, Jun to Aug & $88(33.6)$ & $66(31.6)$ & $22(41.5)$ & & \\
\hline Fall, Sep to Nov & $49(18.7)$ & $42(20.1)$ & $7(13.2)$ & & \\
\hline Winter, Dec to Feb & $68(26.0)$ & $57(27.3)$ & $11(20.8)$ & & \\
\hline Birth length, $\mathrm{cm}$ & $49.96(49.77,50.15)$ & $49.93(49.71,50.16)$ & $50.06(49.69,50.43)$ & -1.07 & 0.291 \\
\hline Birth weight, $\mathrm{kg}$ & $3.31(3.26,3.36)$ & $3.31(3.25,3.37)$ & $3.31(3.23,3.40)$ & -0.58 & 0.563 \\
\hline Birth BMI, $\mathrm{kg} / \mathrm{m}^{2}$ & $13.22(13.08,13.37)$ & $13.24(13.07,13.41)$ & $13.17(12.93,13.41)$ & 0.02 & 0.986 \\
\hline
\end{tabular}


Table 2 Independent effects of WAZ-change trajectories and childhood lifestyle factors on children's adiposity measures at four years old (imputed data).

\begin{tabular}{|c|c|c|c|c|c|c|}
\hline & Weight $\psi$ & BMI & Waist circumference & WHtR & Biceps circumference & Subcutaneous fat \\
\hline & $\beta(95 \% \mathrm{CI})$ & $\beta(95 \% \mathrm{CI})$ & $\beta(95 \% \mathrm{CI})$ & $\beta(95 \% \mathrm{CI})$ & $\beta(95 \% \mathrm{CI})$ & $\beta(95 \% \mathrm{CI})$ \\
\hline \multicolumn{7}{|l|}{ Model 1a-1h $\zeta$} \\
\hline \multicolumn{7}{|l|}{$\mathrm{WAZCT}^{\ddagger}$} \\
\hline Steady & Ref. & Ref. & Ref. & Ref. & Ref. & Ref. \\
\hline Rapid & $0.90(0.37,1.44)^{* * *}$ & $0.93(0.49,1.37) * * *$ & $1.90(0.68,3.12)^{* * *}$ & $0.01(0.00,0.02)$ & $1.05(0.62,1.48)^{* * *}$ & $2.57(1.13,4.01)^{* *}$ \\
\hline FR & $0.46(0.09,0.83)^{*}$ & $0.52(0.22,0.83) * *$ & $1.03(0.22,1.85)^{*}$ & $0.00(0.00,0.01)$ & $0.54(0.25,0.83)^{* * *}$ & $1.41(0.47,2.36)^{* *}$ \\
\hline $\mathrm{EF}$ & $0.71(0.36,1.06)^{* * * *}$ & $0.72(0.44,1.00) * * *$ & $1.65(0.88,2.42)^{* * *}$ & $0.01(0.00,0.02) *$ & $0.69(0.41,0.96)^{* * * *}$ & $1.79(0.87,2.71)^{* * *}$ \\
\hline SR & $-0.65(-1.09,-0.21)^{* *}$ & $-0.69(-1.05,-0.33)^{* * *}$ & $-1.63(-2.58,-0.69)^{* * *}$ & $-0.01(-0.02,0.00)^{*}$ & $-0.60(-0.95,-0.26)^{* *}$ & $-0.90(-2.09,0.29)$ \\
\hline $\mathrm{FF}$ & $-0.51(-0.92,-0.09)^{*}$ & $-0.56(-0.90,-0.21)^{* * *}$ & $-1.19(-2.12,-0.25)^{*}$ & $0.00(-0.01,0.00)$ & $-0.58(-0.91,-0.25)^{* *}$ & $-1.41(-2.53,-0.28) *$ \\
\hline Outdoor playtime & $0.03(-0.08,0.15)$ & $0.01(-0.09,0.10)$ & $-0.15(-0.42,0.13)$ & $0.00(0.00,0.00)$ & $-0.01(-0.11,0.09)$ & $0.09(-0.23,0.40)$ \\
\hline Media time & $0.11(-0.15,0.37)$ & $0.10(-0.12,0.33)$ & $0.28(-0.34,0.90)$ & $0.00(0.00,0.01)$ & $0.26(0.03,0.49)^{*}$ & $0.31(-0.43,1.06)$ \\
\hline Total sleep time & $0.24(-0.10,0.58)$ & $0.22(-0.07,0.52)$ & $0.60(-0.19,1.40)$ & $0.01(0.00,0.01)$ & $0.19(-0.10,0.49)$ & $0.76(-0.20,1.71)$ \\
\hline \multicolumn{7}{|l|}{ Model $2 \xi$} \\
\hline \multicolumn{7}{|l|}{ Model 2a } \\
\hline \multicolumn{7}{|l|}{$\mathrm{WAZCT}^{\ddagger}$} \\
\hline Steady & Ref. & Ref. & Ref. & Ref. & Ref. & Ref. \\
\hline Rapid & $0.83(0.30,1.36)^{* *}$ & $0.82(0.38,1.26)^{* * *}$ & $1.70(0.47,2.92)^{* * *}$ & $0.01(0.00,0.02)$ & $0.92(0.50,1.35)^{* * *}$ & $2.25(0.81,3.69)^{* * *}$ \\
\hline FR & $0.41(0.04,0.78)^{*}$ & $0.44(0.14,0.74)^{* *}$ & $0.88(0.05,1.70) *$ & $0.00(0.00,0.01)$ & $0.44(0.16,0.72)^{* * *}$ & $1.20(0.24,2.15)^{*}$ \\
\hline \multicolumn{7}{|l|}{ Model 2b } \\
\hline \multicolumn{7}{|l|}{ WAZCT $^{\ddagger}$} \\
\hline Steady & Ref. & Ref. & Ref. & Ref. & Ref. & Ref. \\
\hline Rapid & $0.72(0.19,1.24)^{* *}$ & $0.69(0.25,1.12)^{* *}$ & $1.37(0.15,2.59) *$ & $0.01(-0.01,0.02)$ & $0.81(0.39,1.23)^{* * *}$ & $1.96(0.52,3.41)^{* *}$ \\
\hline $\mathrm{EF}$ & $0.64(0.29,1.00)^{* * * *}$ & $0.64(0.35,0.92)^{* * * *}$ & $1.45(0.66,2.25)^{* * *}$ & $0.01(0.00,0.01)$ & $0.60(0.33,0.87)^{* * *}$ & $1.58(0.64,2.53)^{* *}$ \\
\hline \multicolumn{7}{|l|}{ Model 2c } \\
\hline \multicolumn{7}{|l|}{ WAZCT $^{\ddagger}$} \\
\hline Steady & Ref. & Ref. & Ref. & Ref. & Ref. & Ref. \\
\hline Rapid & $0.81(0.28,1.33)^{* *}$ & $0.79(0.35,1.23)^{* * *}$ & $1.58(0.37,2.79)^{*}$ & $0.01(0.00,0.02)$ & $0.92(0.49,1.34)^{* * *}$ & $2.39(0.93,3.85)^{* *}$ \\
\hline SR & $-0.60(-1.05,-0.15)^{*}$ & $-0.62(-0.99,-0.25)^{* *}$ & $-1.50(-2.47,-0.53)^{* * *}$ & $-0.01(-0.02,0.00)$ & $-0.53(-0.87,-0.19)^{* * *}$ & $-0.76(-1.98,0.45)$ \\
\hline \multicolumn{7}{|l|}{ Model 2d } \\
\hline
\end{tabular}




\begin{tabular}{|c|c|c|c|c|c|c|}
\hline Steady & Ref. & Ref. & Ref. & Ref. & Ref. & Ref. \\
\hline Rapid & $0.79(0.25,1.33)^{* *}$ & $0.78(0.33,1.23)^{* *}$ & $1.62(0.37,2.86)^{*}$ & $0.01(0.00,0.02)$ & $0.89(0.45,1.32)^{* * *}$ & $2.21(0.74,3.69)^{* * *}$ \\
\hline $\mathrm{FF}$ & $-0.44(-0.87,-0.01)^{*}$ & $-0.46(-0.82,-0.11)^{*}$ & $-0.97(-1.94,-0.01)$ & $0.00(-0.01,0.01)$ & $-0.48(-0.81,-0.15)^{* * *}$ & $-1.13(-2.29,0.03)$ \\
\hline
\end{tabular}

$\mathrm{BMI}=$ body mass index; $\mathrm{CI}=$ confidence interval; $\mathrm{EF}=$ enjoyment of food; FF = food fussiness; FR = food responsiveness; $\mathrm{SR}=$ satiety responsiveness; WHtR = waist-to-height ratio; WAZCT

$=$ WAZ-change trajectories, i.e. trajectory for change of weight-for-age z-score.

" Adjusted child's height at four years old.

'Examined the effect of WAZ-change trajectory and each interested lifestyle factor on each adiposity measure with adjusting for baseline family income, gestational age of the child at delivery, maternal pre-pregnancy BMI, paternal BMI, newborn weight at the first three days, sex and energy intake at six months. Note, each lifestyle factor was analyzed solitarily, e.g. in Model 1a, we only analyzed WAZ-change trajectory and in Model $1 \mathrm{~b}$ only analyzed food responsiveness (FR) with adjusting for confounders.

รTested the independent effect of WAZ-change trajectory and each childhood lifestyle factors (i.e. four eating behaviors) which reached statistic significance in model 1 , with adjusting for characteristic confounding factors the same as Model 1. Note, four subscales of eating behaviors were analyzed separately, e.g. in Model 2a, both WAZ-change trajectories and food responsiveness (FR) were included in the model to test their independent effects on adiposity indicators.

${ }^{\ddagger}$ Early infancy rapid vs steady WAZ-change trajectory.

$* P<0.05, * * P<0.01, * * * P<0.001$ 
Table 3 Combined effects of WAZ-change trajectories and four subscales of eating behaviors on children's adiposity measures at four years old (imputed data).

\begin{tabular}{|c|c|c|c|c|c|c|}
\hline & Weight $\psi$ & BMI & Waist circumference & WHtR & Biceps circumference & Subcutaneous fat \\
\hline & $\beta(95 \% \mathrm{CI})$ & $\beta(95 \% \mathrm{CI})$ & $\beta(95 \% \mathrm{CI})$ & $\beta(95 \% \mathrm{CI})$ & $\beta(95 \% \mathrm{CI})$ & $\beta(95 \% \mathrm{CI})$ \\
\hline \multicolumn{7}{|l|}{ Model a $\zeta, 1$} \\
\hline Steady \& lower FR & Ref. & Ref. & Ref. & Ref. & Ref. & Ref. \\
\hline Steady \& higher FR & $0.14(-0.70,0.98)$ & $0.24(-0.47,0.94)$ & $0.18(-1.77,2.12)$ & $-0.01(-0.02,0.01)$ & $0.18(-0.49,0.85)$ & $0.96(-1.28,3.20)$ \\
\hline Rapid \& lower FR & $0.62(-0.11,1.35)$ & $0.64(0.02,1.25)$ & $0.94(-0.75,2.63)$ & $0.00(-0.01,0.02)$ & $0.59(0.00,1.18)$ & $1.56(-0.41,3.54)$ \\
\hline Rapid \& higher FR & $1.24(0.51,1.97)^{* *}$ & $1.31(0.73,1.90) * *$ & $2.76(1.15,4.36)^{* *}$ & $0.01(0.00,0.02)$ & $1.49(0.93,2.05)^{* * *}$ & $3.99(2.09,5.89) * * *$ \\
\hline \multicolumn{7}{|l|}{ Model b $\zeta, 1$} \\
\hline Steady \& lower EF & Ref. & Ref. & Ref. & Ref. & Ref. & Ref. \\
\hline Steady \& higher EF & $1.13(0.32,1.95)^{*}$ & $1.08(0.40,1.77)^{*}$ & $2.30(0.41,4.19)$ & $0.02(0.00,0.03)$ & $0.83(0.16,1.49)$ & $2.48(0.28,4.68)$ \\
\hline Rapid \& lower EF & $1.30(0.58,2.02)^{* *}$ & $1.25(0.64,1.85)^{* *}$ & $2.63(0.94,4.32)^{*}$ & $0.02(0.00,0.03)$ & $1.21(0.61,1.81)^{* * *}$ & $3.32(1.32,5.32) * *$ \\
\hline Rapid \& higher EF & $1.43(0.74,2.12)^{* *}$ & $1.46(0.90,2.01)^{* *}$ & $3.02(1.47,4.57)^{* * *}$ & $0.01(0.00,0.03)$ & $1.48(0.94,2.03) * * *$ & $3.73(1.89,5.57) * * *$ \\
\hline \multicolumn{7}{|l|}{ Model c $\zeta, 1$} \\
\hline Steady \& lower SR & $1.04(0.17,1.91)$ & $0.98(0.25,1.71)$ & $1.82(-0.11,3.75)$ & $0.01(0.00,0.03)$ & $0.71(0.03,1.40)$ & $1.44(-0.83,3.71)$ \\
\hline Steady \& higher SR & Ref. & Ref. & Ref. & Ref. & Ref. & Ref. \\
\hline Rapid \& lower SR & $1.60(0.89,2.30)^{* * *}$ & $1.59(1.01,2.17)^{* * *}$ & $3.18(1.57,4.78)^{* * *}$ & $0.02(0.00,0.03)$ & $1.55(0.99,2.12)^{* * *}$ & $3.75(1.83,5.67)^{* * *}$ \\
\hline Rapid \& higher SR & $1.06(0.33,1.78)^{*}$ & $1.04(0.44,1.65)^{* *}$ & $2.09(0.43,3.75)$ & $0.01(0.00,0.03)$ & $1.07(0.49,1.66)^{* * *}$ & $2.44(0.47,4.41)$ \\
\hline \multicolumn{7}{|l|}{ Model d $\zeta, 1$} \\
\hline Steady \& lower FF & $0.33(-0.57,1.24)$ & $0.48(-0.28,1.23)$ & $0.95(-1.07,2.97)$ & $0.00(-0.02,0.02)$ & $0.47(-0.23,1.17)$ & $1.36(-0.95,3.66)$ \\
\hline Steady \& higher FF & Ref. & Ref. & Ref. & Ref. & Ref. & Ref. \\
\hline Rapid \& lower FF & $1.16(0.43,1.90)^{*}$ & $1.24(0.64,1.83)^{* * *}$ & $2.62(1.00,4.24)^{*}$ & $0.01(0.00,0.03)$ & $1.36(0.79,1.93)^{* * *}$ & $3.51(1.58,5.43) * * *$ \\
\hline Rapid \& higher FF & $0.88(0.12,1.64)$ & $0.98(0.35,1.61)^{*}$ & $1.91(0.17,3.64)$ & $0.01(-0.01,0.02)$ & $1.06(0.46,1.66)^{* *}$ & $2.62(0.59,4.65)$ \\
\hline
\end{tabular}

$\mathrm{BMI}=$ body mass index; $\mathrm{CI}=$ confidence interval; $\mathrm{EF}=$ enjoyment of food; $\mathrm{FF}=$ food fussiness; $\mathrm{FR}=$ food responsiveness; $\mathrm{SR}=$ satiety responsiveness; $\mathrm{WHtR}=\mathrm{waist}$-to-height ratio.

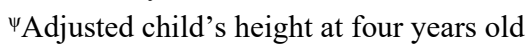

${ }^{\zeta}$ Adjusted for baseline family income, gestational age of the child at delivery, maternal pre-pregnancy BMI, paternal BMI, newborn weight at the first three days, sex and energy intake at six months.

${ }^{1}$ The reference group was children with steady growth trajectory and lower food responsiveness score (model a), lower enjoyment of food score (model b), higher satiety responsiveness score (model c) or higher food fussiness score (model d), which were more likely to have a lower risk of overweight/obesity. Low eating behavior scores present the values less than the median levels of each subscale and the high scores present the values more than the median levels of each subscale.

$* P<0.05, * * P<0.01, * * * P<0.001$ 


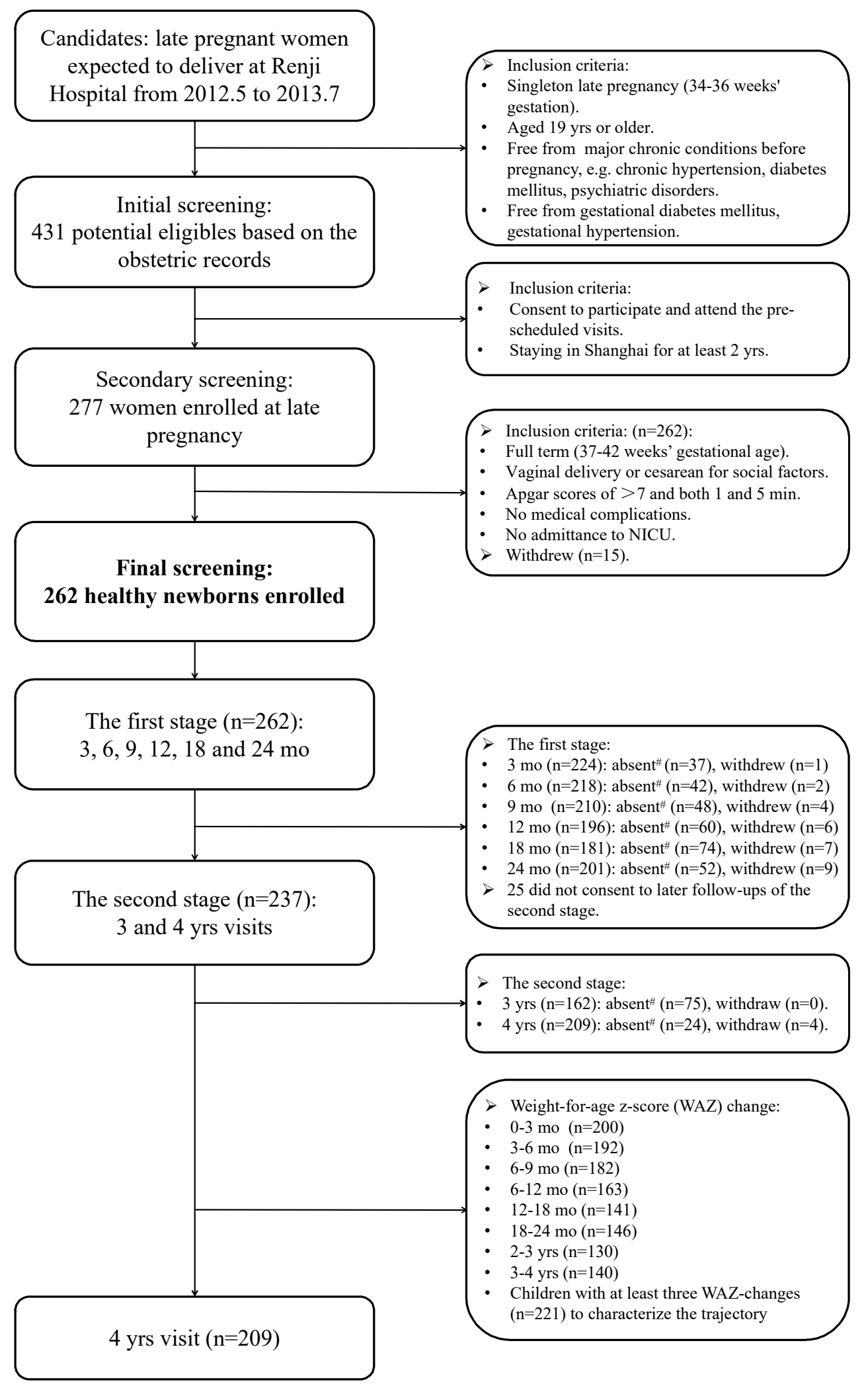

Figure 1. Flow chart of the study participants. "includes non follow-up children and follow-up children with exceeding our critical following-age-window. 


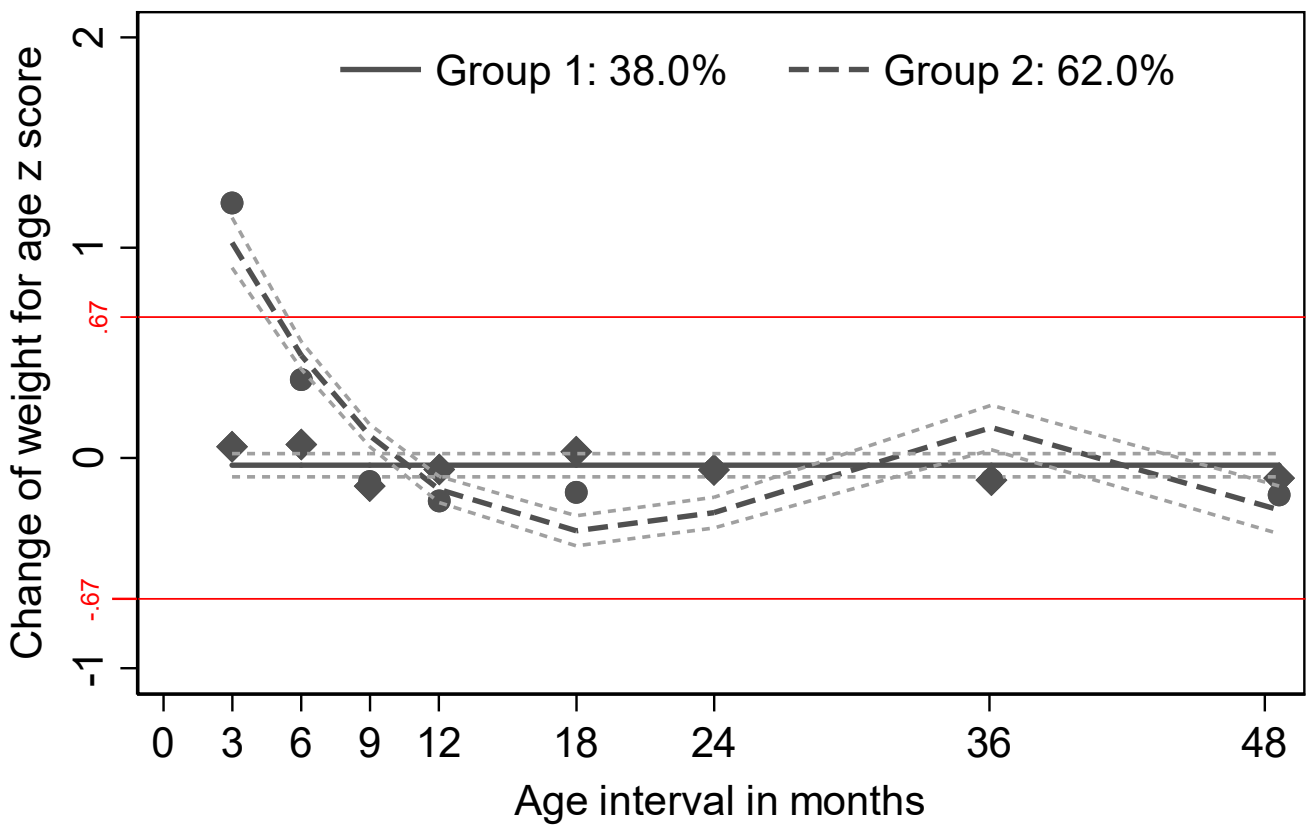

Figure 2. WAZ-change trajectories with $95 \%$ confidence intervals across the first four years.

Note, WAZ-change indicates the change in weight-for-age z-score; the solid line represents steady WAZ-change $(38.0 \%, n=84)$, and the dash line represents early infancy rapid WAZ-change $(62.0 \%, n=137)$. The vertical axis indicates the absolute WAZ-change from the first age point to the second age point with positive values representing WAZ gain and negative values representing WAZ loss, and the +0.67 cut-off means rapid WAZ-change. The horizontal axis indicates the second age point of each WAZ-change month interval, e.g. three-month is the second age point of 0-3 month interval, six-month is the second age point of 3-6 months interval. 

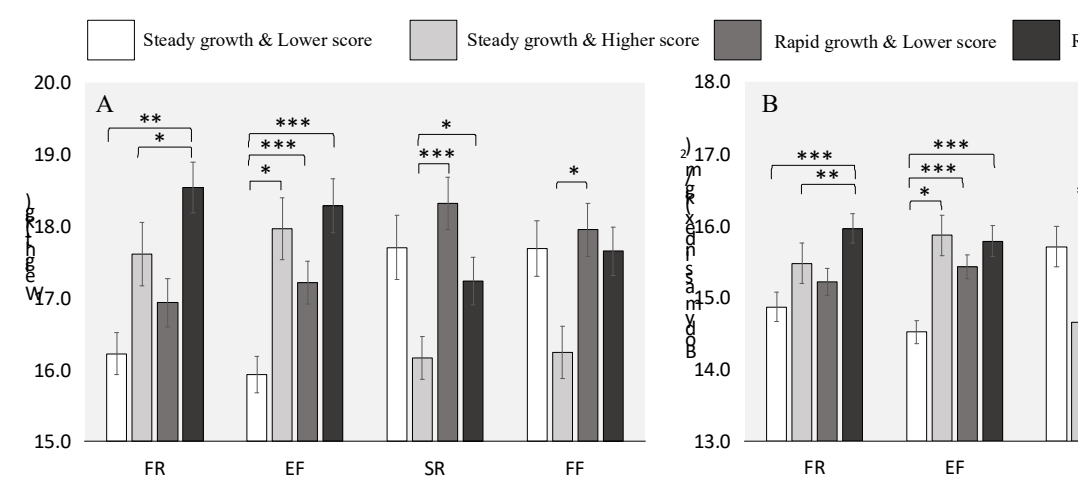

Rapid growth \& Higher score
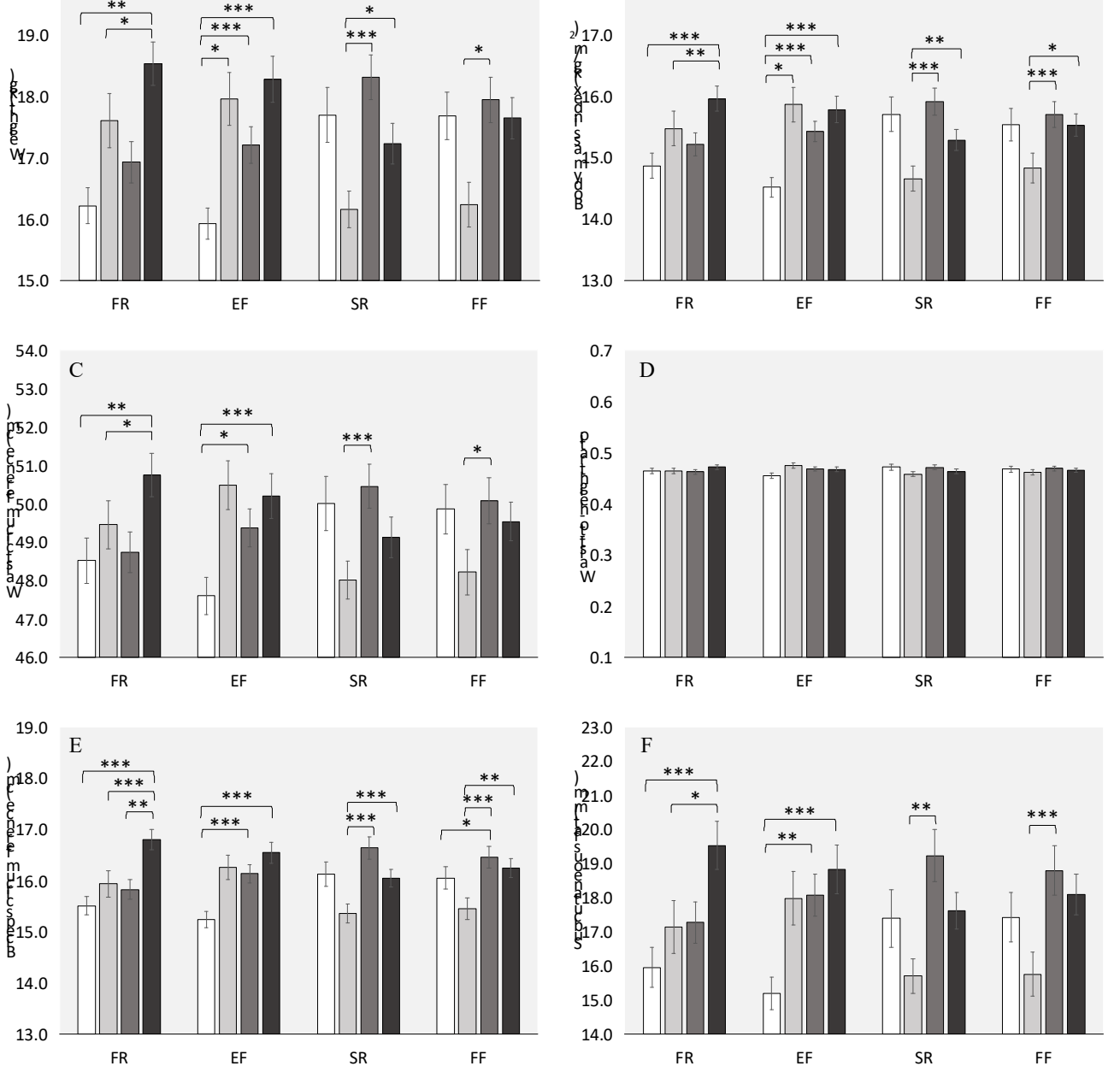

Figure 3. Combined effects of WAZ-change trajectories and four subscales of eating behaviors on children's adiposity measures at four years (imputed data).

Note, EF indicates enjoyment of food; FF indicates food fussiness; FR indicates food responsiveness; SR indicates satiety responsiveness. Low scores present the values less than the median levels of each subscale and the high scores present the values more than the median levels of each subscale.

The Y-axes are: A, weight $(\mathrm{kg})$; B, body mass index $\left(\mathrm{kg} / \mathrm{m}^{2}\right)$; C, waist circumference $(\mathrm{cm})$; D, waist-to-height ratio; $\mathrm{E}$, biceps circumference $(\mathrm{cm}) ; \mathrm{F}$, subcutaneous fat $(\mathrm{mm})$ respectively. The $\mathrm{X}$-axes are the combined groups with the two distinct WAZ-change trajectories and the dichotomy variable of four subscales for eating behaviors (FR, $\mathrm{EF}, \mathrm{SR}$ and FF). The white bars indicate children with steady growth trajectory and lower score of the four eating behaviors, the very light black bars indicate children with steady growth trajectory and higher score of the four eating behaviors, the moderate black bars indicate children with early infancy rapid growth trajectory and lower score of the four eating behaviors, and the dark black bars indicate children with early infancy rapid growth trajectory and higher score of the four eating behaviors. Note, EF indicates enjoyment of food; FF indicates food fussiness; FR indicates food responsiveness; SR indicates satiety responsiveness. Low scores present the values less than the median levels of each subscale and the high scores present the values more than the median levels of each subscale.

Error bars represent mean \pm s.e.m.

$* P<0.05,{ }^{*} P<0.01, * * * P<0.001$. 
Table S1 Absolute WAZ-change and weight increment of the steady and early infancy rapid growth trajectory groups in each age interval (non-imputed data).

\begin{tabular}{|c|c|c|c|c|c|c|c|c|c|}
\hline \multirow{2}{*}{$\begin{array}{c}\text { Age } \\
\text { interval }\end{array}$} & \multirow{2}{*}{$\begin{array}{c}\text { WAZ-change } \\
\geq 0.67 \\
\mathrm{n}(\%)\end{array}$} & \multicolumn{4}{|c|}{ Absolute WAZ-change } & \multicolumn{4}{|c|}{ Weight increment $(\mathrm{kg})$} \\
\hline & & $\begin{array}{c}\text { Total } \\
(\mathrm{n}=221)\end{array}$ & $\begin{array}{l}\text { Steady } \\
(\mathrm{n}=84)\end{array}$ & $\begin{array}{c}\text { Early infancy rapid } \\
(\mathrm{n}=137)\end{array}$ & $P$ value & $\begin{array}{c}\text { Total } \\
(n=221)\end{array}$ & $\begin{array}{l}\text { Steady } \\
(\mathrm{n}=84)\end{array}$ & $\begin{array}{c}\text { Early infancy rapid } \\
(\mathrm{n}=137)\end{array}$ & $P$ value \\
\hline 0-3 mo & $108(54.0)$ & $0.80(0.67,0.93)$ & $0.00(-0.13,0.13)$ & $1.27(1.14,1.40)$ & $<0.001$ & $3.37(3.27,3.46)$ & $2.89(2.78,3.01)$ & $3.65(3.55,3.76)$ & $<0.001$ \\
\hline $3-6 \mathrm{mo}$ & $44(22.9)$ & $0.26(0.19,0.34)$ & $0.01(-0.10,0.12)$ & $0.40(0.31,0.49)$ & $<0.001$ & $1.77(1.69,1.84)$ & $1.49(1.40,1.57)$ & $1.92(1.83,2.02)$ & $<0.001$ \\
\hline $6-9$ mo & $9(5.0)$ & $-0.12(-0.19,-0.05)$ & $-0.16(-0.29,-0.03)$ & $-0.10(-0.18,-0.02)$ & 0.412 & $1.00(0.93,1.07)$ & $0.90(0.79,1.01)$ & $1.06(0.98,1.14)$ & 0.025 \\
\hline $9-12 \mathrm{mo}$ & $6(3.7)$ & $-0.15(-0.22,-0.08)$ & $-0.05(-0.15,0.06)$ & $-0.21(-0.30,-0.12)$ & 0.025 & $0.71(0.63,0.78)$ & $0.78(0.66,0.89)$ & $0.66(0.57,0.76)$ & 0.129 \\
\hline $12-18 \mathrm{mo}$ & $10(7.1)$ & $-0.09(-0.17,-0.01)$ & $0.10(-0.03,0.23)$ & $-0.21(-0.31,-0.11)$ & $<0.001$ & $1.32(1.21,1.42)$ & $1.48(1.31,1.65)$ & $1.22(1.08,1.35)$ & 0.016 \\
\hline $18-24 \mathrm{mo}$ & $7(4.8)$ & $-0.06(-0.13,0.01)$ & $-0.06(-0.17,0.05)$ & $-0.05(-0.15,0.04)$ & 0.896 & $1.27(1.17,1.38)$ & $1.18(1.03,1.32)$ & $1.32(1.18,1.47)$ & 0.193 \\
\hline $2-3 \mathrm{yrs}$ & $5(3.9)$ & $-0.10(-0.18,-0.03)$ & $-0.13(-0.22,-0.04)$ & $-0.09(-0.20,0.03)$ & 0.602 & $2.39(2.22,2.56)$ & $2.11(1.97,2.25)$ & $2.56(2.31,2.82)$ & 0.010 \\
\hline $3-4$ yrs & $4(2.9)$ & $-0.15(-0.21,-0.08)$ & $-0.06(-0.16,0.04)$ & $-0.20(-0.29,-0.11)$ & 0.042 & $2.19(2.04,2.35)$ & $2.24(2.03,2.46)$ & $2.16(1.94,2.37)$ & 0.597 \\
\hline
\end{tabular}

Note, WAZ-change, change of weight-for-age z-score between adjacent measurement points with positive differences representing WAZ gain and negative differences representing WAZ loss. 
Table S2 Maternal-child characteristics between the two identified growth trajectories (non-imputed data).

\begin{tabular}{|c|c|c|c|c|c|}
\hline & $\begin{array}{c}\text { All } \\
(n=221)\end{array}$ & $\begin{array}{l}\text { Steady } \\
(n=84)\end{array}$ & $\begin{array}{c}\text { Rapid } \\
(\mathrm{n}=137)\end{array}$ & $\mathbf{t} / \chi^{2}$ & $P$ value \\
\hline \multicolumn{6}{|l|}{ Demographic Factors } \\
\hline Highest parents education & & & & 0.69 & 0.709 \\
\hline High school or lower & $11(5.0)$ & $3(3.6)$ & $8(5.8)$ & & \\
\hline College or higher & $210(95.0)$ & $81(96.4)$ & $129(94.2)$ & & \\
\hline Maternal age at birth, year & $29.47(29.04,29.90)$ & $29.60(28.81,30.39)$ & $29.39(28.88,29.90)$ & -0.47 & 0.642 \\
\hline Gestational age, week & $39.59(39.46,39.73)$ & $39.89(39.70,40.09)$ & $39.41(39.24,39.58)$ & -3.66 & $<0.001$ \\
\hline Pre-pregnancy BMI, kg/m² & $20.60(20.25,20.95)$ & $20.78(20.13,21.42)$ & $20.49(20.08,20.90)$ & -0.78 & 0.436 \\
\hline Paternal BMI, $\mathrm{kg} / \mathrm{m}^{2}$ & $24.52(23.95,25.10)$ & $24.92(24.22,25.63)$ & $24.28(23.45,25.10)$ & -1.07 & 0.284 \\
\hline Gestational weight gain, $\mathrm{kg}$ & $16.16(15.58,16.74)$ & $16.50(15.50,17.50)$ & $15.95(15.24,16.67)$ & -0.90 & 0.368 \\
\hline Maternal sleep quality & $85(42.1)$ & $30(39.5)$ & $55(43.7)$ & 0.34 & 0.560 \\
\hline Maternal state anxiety & $18(8.7)$ & $7(9.0)$ & $11(8.5)$ & 0.01 & 0.912 \\
\hline Maternal trait anxiety & $28(13.6)$ & $12(15.8)$ & $16(12.3)$ & 0.50 & 0.482 \\
\hline Maternal depression & $27(12.7)$ & $9(11.1)$ & $18(13.6)$ & 0.29 & 0.591 \\
\hline \multicolumn{6}{|l|}{ Newborn } \\
\hline Male & $113(51.1)$ & $45(53.6)$ & $68(49.6)$ & 0.32 & 0.570 \\
\hline Vaginal delivery & $85(39.7)$ & $28(35.4)$ & $57(42.2)$ & 0.96 & 0.328 \\
\hline Birth length, $\mathrm{cm}$ & $49.92(49.7,50.13)$ & $50.28(49.92,50.63)$ & $49.69(49.43,49.95)$ & -2.68 & 0.008 \\
\hline Birth weight, kg & $3.30(3.25,3.35)$ & $3.48(3.40,3.56)$ & $3.19(3.13,3.25)$ & -5.64 & $<0.001$ \\
\hline Birth BMI, $\mathrm{kg} / \mathrm{m}^{2}$ & $13.22(13.06,13.37)$ & $13.74(13.49,14.00)$ & $12.89(12.71,13.06)$ & -5.67 & $<0.001$ \\
\hline \multicolumn{6}{|l|}{ Infancy } \\
\hline $\begin{array}{l}\text { Exclusive breastfeeding over the } \\
\text { first three months, Yes }\end{array}$ & $88(40.2)$ & $37(45.1)$ & $51(37.2)$ & 1.33 & 0.249 \\
\hline Weaning time & & & & 1.23 & 0.268 \\
\hline$<6$ months & $94(43.1)$ & $39(46.4)$ & $55(41.0)$ & & \\
\hline 6-12 months & $71(32.6)$ & $29(34.5)$ & $42(31.3)$ & & \\
\hline$\geq 12$ months & $47(29.2)$ & $20(34.5)$ & $27(26.2)$ & & \\
\hline $\begin{array}{l}\text { Total energy intake at } 6 \text { months, } \\
\text { kcal/day }\end{array}$ & $\begin{array}{c}580.26 \\
(558.45,602.07)\end{array}$ & $\begin{array}{c}573.78 \\
(541.8,605.77)\end{array}$ & $\begin{array}{c}584.14 \\
(554.68,613.60)\end{array}$ & 0.45 & 0.652 \\
\hline \multicolumn{6}{|l|}{ Lifestyle in childhood } \\
\hline \multicolumn{6}{|l|}{ Eating behaviors ${ }^{a}$} \\
\hline FR, unit & $2.76(2.64,2.89)$ & $2.67(2.48,2.86)$ & $2.83(2.66,2.99)$ & 1.25 & 0.215 \\
\hline $\mathrm{EF}$, unit & $3.59(3.47,3.71)$ & $3.43(3.23,3.64)$ & $3.70(3.55,3.85)$ & 2.16 & $\mathbf{0 . 0 3 3}$ \\
\hline SR, unit & $2.66(2.56,2.76)$ & $2.73(2.57,2.89)$ & $2.61(2.48,2.74)$ & -1.18 & 0.240 \\
\hline $\mathrm{FF}$, unit & $2.67(2.56,2.78)$ & $2.80(2.65,2.96)$ & $2.58(2.43,2.73)$ & -2.03 & 0.044 \\
\hline Outdoor playtime, hour/day & $3.94(3.63,4.25)$ & $4.41(3.84,4.97)$ & $3.63(3.28,3.99)$ & -2.42 & 0.016 \\
\hline Media time, hour/day ${ }^{a}$ & $1.40(1.25,1.54)$ & $1.34(1.07,1.60)$ & $1.43(1.24,1.61)$ & 0.57 & 0.572 \\
\hline Total sleep time, hour/day ${ }^{a}$ & $11.98(11.88,12.08)$ & $11.95(11.80,12.11)$ & $11.99(11.85,12.14)$ & 0.37 & 0.711 \\
\hline \multicolumn{6}{|l|}{ Adiposity outcomes at 4 years } \\
\hline Height, cm & $\begin{array}{c}106.09 \\
(105.51,106.67)\end{array}$ & $\begin{array}{c}105.38 \\
(104.51,106.25)\end{array}$ & $\begin{array}{c}106.58 \\
(105.81,107.35)\end{array}$ & 2.02 & 0.045 \\
\hline Weight, kg & $17.42(17.06,17.78)$ & $16.82(16.32,17.33)$ & $17.83(17.34,18.31)$ & 2.77 & 0.006 \\
\hline $\mathrm{BMI}, \mathrm{kg} / \mathrm{m}^{2}$ & $15.42(15.21,15.63)$ & $15.11(14.78,15.43)$ & $15.63(15.36,15.91)$ & 2.44 & 0.016 \\
\hline Waist circumference, $\mathrm{cm}$ & $49.44(48.88,50.00)$ & $48.86(48.06,49.66)$ & $49.85(49.08,50.61)$ & 1.72 & 0.087 \\
\hline WHtR, unit & $0.47(0.46,0.47)$ & $0.46(0.46,0.47)$ & $0.47(0.46,0.47)$ & 0.78 & 0.438 \\
\hline Biceps circumference, $\mathrm{cm}$ & $16.1(15.90,16.31)$ & $15.70(15.42,15.99)$ & $16.38(16.10,16.66)$ & 3.28 & 0.001 \\
\hline Subcutaneous fat, $\mathrm{mm}$ & $17.71(17.04,18.38)$ & $16.50(15.59,17.41)$ & $18.54(17.62,19.46)$ & 3.02 & 0.003 \\
\hline
\end{tabular}

$\mathrm{BMI}=$ body mass index; $\mathrm{CI}=$ confidence interval; $\mathrm{EF}=$ enjoyment of food; $\mathrm{FF}=$ Food fussiness; $\mathrm{FR}=$ food responsiveness;

$\mathrm{SR}=$ satiety responsiveness; $\mathrm{WHtR}=$ waist-to-height ratio; $\mathrm{WAZ}=$ weight-for-age $\mathrm{z}$-score.

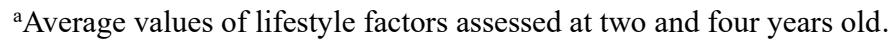


Table S3 Interactive effects of WAZ-change trajectories and four subscales of eating behaviors on children's adiposity measures at four years (imputed data).

\begin{tabular}{|c|c|c|c|c|c|c|}
\hline & Weight $\psi$ & BMI & Waist circumference & WHtR & Biceps circumference & Subcutaneous fat \\
\hline & $\beta(95 \% \mathrm{CI})$ & $\beta(95 \% \mathrm{CI})$ & $\beta(95 \% \mathrm{CI})$ & $\beta(95 \% \mathrm{CI})$ & $\beta(95 \% \mathrm{CI})$ & $\beta(95 \% \mathrm{CI})$ \\
\hline \multicolumn{7}{|l|}{ Model 1a } \\
\hline \multicolumn{7}{|l|}{$\mathrm{WAZCT}^{\ddagger}$} \\
\hline Steady & Ref. & Ref. & Ref. & Ref. & Ref. & Ref. \\
\hline Rapid & $0.55(-0.18,1.27)$ & $0.56(-0.04,1.17)$ & $0.82(-0.84,2.48)$ & $0(-0.01,0.02)$ & $0.51(-0.07,1.09)$ & $1.47(-0.47,3.41)$ \\
\hline \multicolumn{7}{|l|}{$\mathrm{FR}^{\zeta}$} \\
\hline Low & Ref. & Ref. & Ref. & Ref. & Ref. & Ref. \\
\hline High & $0.25(-0.57,1.07)$ & $0.33(-0.36,1.01)$ & $0.31(-1.57,2.20)$ & $0(-0.02,0.01)$ & $0.23(-0.42,0.88)$ & $1.17(-1.00,3.34)$ \\
\hline WAZCT*FR & $0.48(-0.54,1.50)$ & $0.43(-0.43,1.29)$ & $1.66(-0.70,4.02)$ & $0.01(-0.01,0.04)$ & $0.73(-0.09,1.54)$ & $1.37(-1.40,4.13)$ \\
\hline \multicolumn{7}{|l|}{ Model 1b } \\
\hline \multicolumn{7}{|l|}{$\mathrm{WAZCT}^{\ddagger}$} \\
\hline Steady & Ref. & Ref. & Ref. & Ref. & Ref. & Ref. \\
\hline Rapid & $1.25(0.54,1.96)^{* *}$ & $1.18(0.59,1.77)^{* * *}$ & $2.52(0.87,4.16)^{* *}$ & $0.02(0,0.03)^{*}$ & $1.12(0.54,1.70)^{* * *}$ & $3.26(1.31,5.21)^{* * *}$ \\
\hline \multicolumn{7}{|l|}{$\mathrm{EF}^{\zeta}$} \\
\hline Low & Ref. & Ref. & Ref. & Ref. & Ref. & Ref. \\
\hline High & $1.20(0.41,1.99)^{* *}$ & $1.13(0.47,1.79)^{* * *}$ & $2.35(0.53,4.17)^{*}$ & $0.02(0,0.03)^{*}$ & $0.84(0.20,1.49)^{*}$ & $2.72(0.60,4.84)^{*}$ \\
\hline WAZCT*FR & $-0.98(-1.99,0.03)$ & $-0.86(-1.71,-0.02)^{*}$ & $-1.83(-4.17,0.52)$ & $-0.02(-0.04,0)$ & $-0.52(-1.36,0.31)$ & $-2.16(-4.95,0.64)$ \\
\hline \multicolumn{7}{|l|}{ Model 1c } \\
\hline \multicolumn{7}{|l|}{$\mathrm{WAZCT}^{\ddagger}$} \\
\hline Steady & Ref. & Ref. & Ref. & Ref. & Ref. & Ref. \\
\hline Rapid & $0.56(-0.21,1.32)$ & $0.58(-0.06,1.22)$ & $1.29(-0.43,3.01)$ & $0(-0.01,0.02)$ & $0.82(0.21,1.43)^{* *}$ & $2.35(0.28,4.41)^{*}$ \\
\hline \multicolumn{7}{|l|}{$\mathrm{SR} \zeta$} \\
\hline Low & Ref. & Ref. & Ref. & Ref. & Ref. & Ref. \\
\hline High & $-1.01(-1.86,-0.17)^{*}$ & $-0.94(-1.65,-0.24)^{* * *}$ & $-1.88(-3.73,-0.02)^{*}$ & $-0.01(-0.03,0)$ & $-0.64(-1.31,0.03)$ & $-1.32(-3.51,0.88)$ \\
\hline WAZCT*FR & $0.41(-0.64,1.46)$ & $0.35(-0.54,1.24)$ & $0.69(-1.65,3.04)$ & $0.01(-0.01,0.03)$ & $0.12(-0.73,0.95)$ & $-0.14(-2.96,2.68)$ \\
\hline \multicolumn{7}{|l|}{ Model 1d } \\
\hline \multicolumn{7}{|l|}{$\mathrm{WAZCT}^{\ddagger}$} \\
\hline Steady & Ref. & Ref. & Ref. & Ref. & Ref. & Ref. \\
\hline Rapid & $0.79(0.03,1.56)^{*}$ & $0.71(0.06,1.36)^{*}$ & $1.56(-0.19,3.31)$ & $0.01(-0.01,0.03)$ & $0.86(0.24,1.47)^{* *}$ & $2.14(0.09,4.19)^{*}$ \\
\hline \multicolumn{7}{|l|}{$\mathrm{FF} \zeta$} \\
\hline Low & Ref. & Ref. & Ref. & Ref. & Ref. & Ref. \\
\hline High & $-0.35(-1.23,0.53)$ & $-0.47(-1.20,0.27)$ & $-1.05(-3.00,0.91)$ & $0(-0.02,0.02)$ & $-0.41(-1.09,0.27)$ & $-1.28(-3.52,0.96)$ \\
\hline
\end{tabular}




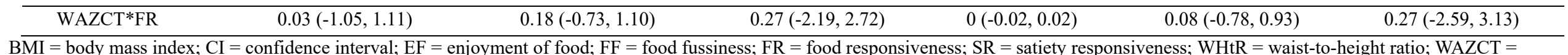

$\mathrm{BMI}=$ body mass index; $\mathrm{CI}=$ confidence interval; $\mathrm{EF}=$ enjoyment of food; $\mathrm{FF}=$ food fussiness; $\mathrm{FR}=$ food responsiveness; $\mathrm{SR}=$ satiety responsiveness; $\mathrm{WHtR}=$ waist-to-height ratio; $\mathrm{WAZCT}=$

WAZ-change trajectories, i.e. trajectory for change of weight-for-age z-score.

${ }^{\psi}$ Adjusted children's height at four years old.

$\zeta$ Low scores present the values less than the median levels of each subscale and the high scores present the values more than the median levels of each subscale.

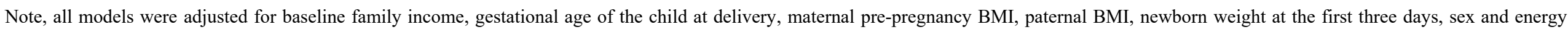
intake at six months.

$* P<0.05, * * P<0.01, * * * P<0.001$ 
Table S4 Independent effects of WAZ-change trajectories and childhood lifestyle factors on children's adiposity measures at four years (non imputed data).

\begin{tabular}{|c|c|c|c|c|c|c|}
\hline & Weight ${ }^{\psi}$ & BMI & Waist circumference & WHtR & Biceps circumference & Subcutaneous fat \\
\hline & $\beta(95 \% \mathrm{CI})$ & $\beta(95 \% \mathrm{CI})$ & $\beta(95 \% \mathrm{CI})$ & $\beta(95 \% \mathrm{CI})$ & $\beta(95 \% \mathrm{CI})$ & $\beta(95 \% \mathrm{CI})$ \\
\hline \multicolumn{7}{|l|}{ Model 1a-1h $\zeta$} \\
\hline \multicolumn{7}{|l|}{$\mathrm{WAZCT}^{\ddagger}$} \\
\hline Steady & Ref. & Ref. & Ref. & Ref. & Ref. & Ref. \\
\hline Rapid & $0.86(0.30,1.43)^{* *}$ & $0.89(0.42,1.35)^{* * *}$ & $2.07(0.81,3.33)^{* *}$ & $0.01(0.02,0.15)$ & $1.00(0.56,1.44)^{* * *}$ & $2.16(0.62,3.7)^{* *}$ \\
\hline FR & $0.63(0.27,0.99) * *$ & $0.63(0.32,0.93)^{* * *}$ & $1.59(0.74,2.44)^{* * *}$ & $0.01(0.02,0.19)$ & $0.67(0.35,0.99)^{* * *}$ & $1.56(0.46,2.65)^{* *}$ \\
\hline $\mathrm{EF}$ & $0.59(0.24,0.93) * * *$ & $0.57(0.28,0.86)^{* * *}$ & $1.35(0.53,2.17)^{* *}$ & $0.01(0.01,0.15)$ & $0.50(0.19,0.81)^{* *}$ & $1.39(0.34,2.45)^{*}$ \\
\hline SR & $-0.30(-0.73,0.13)$ & $-0.34(-0.71,0.03)$ & $-0.73(-1.79,0.32)$ & $0.00(0.01,0.00)$ & $-0.30(-0.69,0.10)$ & $0.04(-1.32,1.4)$ \\
\hline $\mathrm{FF}$ & $-0.42(-0.82,-0.02)^{*}$ & $-0.45(-0.79,-0.11)^{*}$ & $-0.79(-1.76,0.18)$ & $0.00(0.01,-0.03)$ & $-0.35(-0.72,0.02)$ & $-1.12(-2.34,0.11)$ \\
\hline Outdoor playtime & $0.03(-0.09,0.15)$ & $0.00(-0.10,0.11)$ & $-0.15(-0.42,0.12)$ & $0.00(0.00,-0.04)$ & $-0.02(-0.12,0.08)$ & $0.09(-0.25,0.43)$ \\
\hline Media time & $0.07(-0.28,0.42)$ & $0.09(-0.22,0.39)$ & $0.33(-0.49,1.14)$ & $0.00(0.01,0.01)$ & $0.31(0.01,0.60)^{*}$ & $0.4(-0.61,1.42)$ \\
\hline Total sleep time & $0.3(-0.05,0.66)$ & $0.28(-0.04,0.59)$ & $0.85(0.01,1.69)^{*}$ & $0.01(0.01,0.16)$ & $0.24(-0.07,0.55)$ & $0.97(-0.05,1.98)$ \\
\hline \multicolumn{7}{|l|}{ Model $2 \xi$} \\
\hline \multicolumn{7}{|l|}{ Model 2a } \\
\hline \multicolumn{7}{|l|}{$\mathrm{WAZCT}^{\ddagger}$} \\
\hline Steady & Ref. & Ref. & Ref. & Ref. & Ref. & Ref. \\
\hline Rapid & $0.81(0.25,1.36)^{* *}$ & $0.76(0.29,1.23)^{* *}$ & $1.64(0.31,2.98)^{*}$ & $0.01(0.02,0.15)$ & $0.83(0.34,1.32)^{* *}$ & $2.36(0.67,4.05)^{* * *}$ \\
\hline FR & $0.59(0.23,0.95)^{* *}$ & $0.56(0.26,0.86) * * *$ & $1.46(0.61,2.32)^{* *}$ & $0.01(0.02,0.18)$ & $0.58(0.27,0.89) * * *$ & $1.35(0.27,2.42)^{*}$ \\
\hline \multicolumn{7}{|l|}{ Model 2b } \\
\hline \multicolumn{7}{|l|}{$\mathrm{WAZCT}^{\ddagger}$} \\
\hline Steady & Ref. & Ref. & Ref. & Ref. & Ref. & Ref. \\
\hline Rapid & $0.73(0.18,1.28)^{*}$ & $0.67(0.20,1.14)^{* *}$ & $1.36(-0.01,2.73)$ & $0.01(0.02,0.13)$ & $0.76(0.26,1.25)^{* * *}$ & $2.13(0.43,3.83)^{*}$ \\
\hline EF & $0.55(0.19,0.90)^{* *}$ & $0.51(0.22,0.80)^{* *}$ & $1.20(0.35,2.06)^{* *}$ & $0.00(0.01,0.11)$ & $0.46(0.15,0.77)^{* *}$ & $1.24(0.18,2.29)^{*}$ \\
\hline \multicolumn{7}{|l|}{ Model 2c } \\
\hline \multicolumn{7}{|l|}{$\mathrm{WAZCT}^{\ddagger}$} \\
\hline Steady & Ref. & Ref. & Ref. & Ref. & Ref. & Ref. \\
\hline Rapid & $0.89(0.33,1.45)^{* *}$ & $0.82(0.35,1.30)^{* *}$ & $1.73(0.32,3.14)^{*}$ & $0.01(0.02,0.16)$ & $0.89(0.39,1.40)^{* *}$ & $2.61(0.85,4.37)^{* *}$ \\
\hline SR & $-0.32(-0.76,0.12)$ & $-0.34(-0.70,0.03)$ & $-0.72(-1.8,0.35)$ & $0.00(0.01,0.02)$ & $-0.31(-0.70,0.08)$ & $-0.05(-1.4,1.3)$ \\
\hline \multicolumn{7}{|l|}{ Model 2d } \\
\hline \multicolumn{7}{|l|}{$\mathrm{WAZCT}^{\ddagger}$} \\
\hline Steady & Ref. & Ref. & Ref. & Ref. & Ref. & Ref. \\
\hline Rapid & $0.80(0.22,1.37)^{* *}$ & $0.75(0.26,1.24) * *$ & $1.54(0.13,2.96)^{*}$ & $0.01(0.02,0.16)$ & $0.82(0.31,1.33)^{* *}$ & $2.3(0.58,4.02)^{* * *}$ \\
\hline
\end{tabular}


WAZ-change trajectories, i.e. trajectory for change of weight-for-age z-score.

${ }^{\psi}$ Adjusted children's height at four years old.

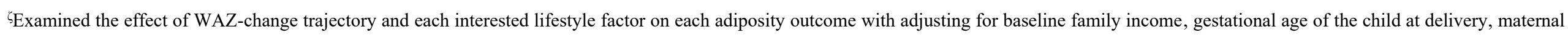

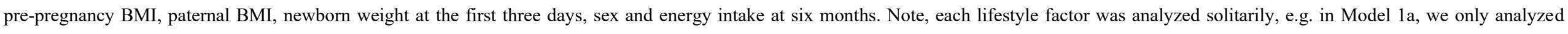
WAZ-change trajectory with adjusting for confounders and in Model $1 \mathrm{~b}$ only analyzed food responsiveness (FR) with adjusting for confounders.

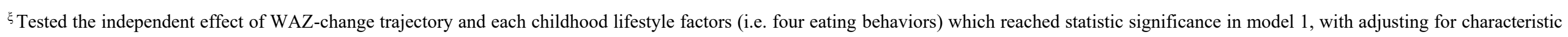

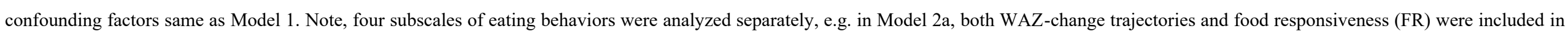
the model to test their independent effects on adiposity indicators.

${ }^{\ddagger}$ Early infancy rapid vs steady WAZ-change trajectory.

$* P<0.05, * * P<0.01, * * * P<0.001$ 
Table S5 Combined effects of WAZ-change trajectories and four subscales of eating behaviors on children's adiposity measures at four years (non imputed data).

\begin{tabular}{|c|c|c|c|c|c|c|}
\hline & Weight $\psi$ & BMI & Waist circumference & WHtR & Biceps circumference & Subcutaneous fat \\
\hline & $\beta(95 \% \mathrm{CI})$ & $\beta(95 \% \mathrm{CI})$ & $\beta(95 \% \mathrm{CI})$ & $\beta(95 \% \mathrm{CI})$ & $\beta(95 \% \mathrm{CI})$ & $\beta(95 \% \mathrm{CI})$ \\
\hline \multicolumn{7}{|l|}{ Model a $\zeta, 1$} \\
\hline Steady \& lower FR & Ref. & Ref. & Ref. & Ref. & Ref. & Ref. \\
\hline Steady \& higher FR & $0.13(-0.71,0.96)$ & $0.14(-0.57,0.86)$ & $0.23(-1.78,2.24)$ & $-0.01(-0.03,0.01)$ & $-0.09(-0.80,0.63)$ & $-0.04(-2.45,2.38)$ \\
\hline Rapid \& lower FR & $0.61(-0.14,1.36)$ & $0.57(-0.07,1.22)$ & $0.93(-0.87,2.74)$ & $0.00(-0.02,0.02)$ & $0.37(-0.28,1.01)$ & $1.00(-1.17,3.17)$ \\
\hline Rapid \& higher FR & $1.30(0.51,2.09)^{* *}$ & $1.29(0.64,1.94)^{* * *}$ & $3.14(1.31,4.97)^{* *}$ & $0.01(0.00,0.03)$ & $1.46(0.81,2.11)^{* * *}$ & $4.34(2.12,6.57)^{* *}$ \\
\hline \multicolumn{7}{|l|}{ Model b $\zeta, 1$} \\
\hline Steady \& lower EF & Ref. & Ref. & Ref. & Ref. & Ref. & Ref. \\
\hline Steady \& higher EF & $0.73(-0.11,1.56)$ & $0.66(-0.06,1.38)$ & $1.94(-0.12,4.00)$ & $0.01(-0.01,0.03)$ & $0.48(-0.27,1.23)$ & $1.82(-0.73,4.36)$ \\
\hline Rapid \& lower EF & $0.93(0.24,1.62)$ & $0.88(0.28,1.47)^{*}$ & $1.90(0.19,3.60)$ & $0.01(0.00,0.03)$ & $0.86(0.23,1.48)^{*}$ & $2.73(0.63,4.84)$ \\
\hline Rapid \& higher EF & $1.34(0.61,2.07)^{* * *}$ & $1.26(0.65,1.87)^{* * * *}$ & $2.96(1.22,4.70)^{* *}$ & $0.01(0.00,0.03)$ & $1.30(0.66,1.94)^{* * *}$ & $3.55(1.38,5.72)^{* * *}$ \\
\hline \multicolumn{7}{|l|}{ Model c $\zeta, 1$} \\
\hline Steady \& lower SR & $0.72(-0.08,1.53)$ & $0.67(-0.02,1.36)$ & $0.88(-1.19,2.95)$ & $0.00(-0.02,0.02)$ & $0.53(-0.21,1.28)$ & $0.58(-1.97,3.13)$ \\
\hline Steady \& higher SR & Ref. & Ref. & Ref. & Ref. & Ref. & Ref. \\
\hline Rapid \& lower SR & $1.46(0.71,2.21)^{* * *}$ & $1.38(0.75,2.02)^{* * *}$ & $2.60(0.70,4.50)^{*}$ & $0.01(0.00,0.03)$ & $1.28(0.60,1.96)^{* * *}$ & $3.48(1.12,5.83)^{*}$ \\
\hline Rapid \& higher SR & $1.04(0.31,1.76)^{*}$ & $0.95(0.33,1.57)^{*}$ & $1.84(-0.01,3.69)$ & $0.01(-0.01,0.03)$ & $1.05(0.39,1.72)^{*}$ & $2.37(0.09,4.64)$ \\
\hline \multicolumn{7}{|l|}{ Model d $\zeta, 1$} \\
\hline Steady \& lower FF & $0.72(-0.12,1.56)$ & $0.78(0.08,1.48)$ & $1.89(-0.12,3.89)$ & $0.01(-0.01,0.02)$ & $0.69(-0.04,1.42)$ & $2.38(-0.04,4.80)$ \\
\hline Steady \& higher FF & Ref. & Ref. & Ref. & Ref. & Ref. & Ref. \\
\hline Rapid \& lower FF & $1.17(0.42,1.91)^{*}$ & $1.17(0.55,1.79)^{* *}$ & $2.29(0.52,4.06)$ & $0.01(-0.01,0.03)$ & $1.14(0.50,1.79)^{* * *}$ & $3.70(1.53,5.86)^{* * *}$ \\
\hline Rapid \& higher FF & $1.24(0.45,2.04) *$ & $1.19(0.53,1.86)^{* *}$ & $2.80(0.90,4.70)^{*}$ & $0.01(0.00,0.03)$ & $1.25(0.56,1.95)^{* *}$ & $3.32(1.02,5.62)^{*}$ \\
\hline
\end{tabular}

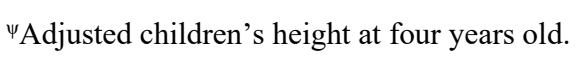

${ }_{5}$ Adjusted for baseline family income, gestational age of the child at delivery, maternal pre-pregnancy BMI, paternal BMI, newborn weight at the first three days, sex and energy intake at six months.

${ }^{1}$ The reference group was children with steady growth trajectory and lower food responsiveness score (model a), lower enjoyment of food score (model b), higher satiety responsiveness score (model c) or higher food fussiness score (model d), which were more likely to have a lower risk of overweight/obesity. Low eating behavior scores present the values less than the median levels of each subscale and the high scores present the values more than the median levels of each subscale.

$* P<0.05, * * P<0.01, * * * P<0.001$. 
Table S6 Interactive effects of WAZ-change trajectories and four subscales of eating behaviors on children's adiposity measures at four years (non imputed data).

\begin{tabular}{|c|c|c|c|c|c|c|}
\hline & Weight $\psi$ & BMI & Waist circumference & WHtR & Biceps circumference & Subcutaneous fat \\
\hline & $\beta(95 \% \mathrm{CI})$ & $\beta(95 \% \mathrm{CI})$ & $\beta(95 \% \mathrm{CI})$ & $\beta(95 \% \mathrm{CI})$ & $\beta(95 \% \mathrm{CI})$ & $\beta(95 \% \mathrm{CI})$ \\
\hline \multicolumn{7}{|l|}{ Model 1a } \\
\hline \multicolumn{7}{|l|}{$\mathrm{WAZCT}^{\ddagger}$} \\
\hline Steady & Ref. & Ref. & Ref. & Ref. & Ref. & Ref. \\
\hline Rapid & $0.55(-0.18,1.29)$ & $0.52(-0.10,1.15)$ & $0.89(-0.85,2.63)$ & $0(-0.02,0.02)$ & $0.29(-0.34,0.93)$ & $1.03(-1.11,3.18)$ \\
\hline \multicolumn{7}{|l|}{$\mathrm{FR}^{\zeta}$} \\
\hline Low & Ref. & Ref. & Ref. & Ref. & Ref. & Ref. \\
\hline High & $0.23(-0.58,1.03)$ & $0.24(-0.45,0.92)$ & $0.33(-1.57,2.22)$ & $-0.01(-0.02,0.01)$ & $-0.03(-0.72,0.66)$ & $0.16(-2.17,2.50)$ \\
\hline WAZCT*FR & $0.55(-0.50,1.60)$ & $0.54(-0.37,1.45)$ & $1.97(-0.54,4.48)$ & $0.02(0,0.04)$ & $1.17(0.25,2.08)^{*}$ & $3.25(0.14,6.35)^{*}$ \\
\hline \multicolumn{7}{|l|}{ Model 1b } \\
\hline \multicolumn{7}{|l|}{$\mathrm{WAZCT}^{\ddagger}$} \\
\hline Steady & Ref. & Ref. & Ref. & Ref. & Ref. & Ref. \\
\hline Rapid & $0.91(0.25,1.58)^{* * *}$ & $0.85(0.27,1.42)^{* * *}$ & $1.89(0.28,3.50)^{*}$ & $0.01(0,0.03)$ & $0.8(0.20,1.39)^{* * *}$ & $2.92(0.89,4.95)^{* * *}$ \\
\hline \multicolumn{7}{|l|}{$\mathrm{EF}^{\zeta}$} \\
\hline Low & Ref. & Ref. & Ref. & Ref. & Ref. & Ref. \\
\hline High & $0.82(0.03,1.61)^{*}$ & $0.74(0.06,1.42)^{*}$ & $1.94(0.03,3.86)^{*}$ & $0.01(-0.01,0.03)$ & $0.54(-0.17,1.25)$ & $2.20(-0.21,4.62)$ \\
\hline WAZCT*FR & $-0.32(-1.34,0.70)$ & $-0.29(-1.18,0.59)$ & $-0.78(-3.27,1.72)$ & $-0.01(-0.03,0.02)$ & $-0.04(-0.97,0.88)$ & $-1.29(-4.44,1.87)$ \\
\hline \multicolumn{7}{|l|}{ Model 1c } \\
\hline \multicolumn{7}{|l|}{ WAZCT $^{\ddagger}$} \\
\hline Steady & Ref. & Ref. & Ref. & Ref. & Ref. & Ref. \\
\hline Rapid & $0.81(0.04,1.59) *$ & $0.77(0.10,1.44)^{*}$ & $1.79(-0.17,3.75)$ & $0.01(-0.01,0.03)$ & $0.8(0.07,1.52)^{*}$ & $3.21(0.72,5.69) *$ \\
\hline \multicolumn{7}{|l|}{$\mathrm{SR}^{\zeta}$} \\
\hline Low & Ref. & Ref. & Ref. & Ref. & Ref. & Ref. \\
\hline High & $-0.64(-1.41,0.13)$ & $-0.58(-1.24,0.09)$ & $-0.89(-2.83,1.06)$ & $0(-0.02,0.02)$ & $-0.41(-1.13,0.30)$ & $-0.41(-2.86,2.04)$ \\
\hline WAZCT*FR & $0.12(-0.89,1.13)$ & $0.07(-0.80,0.95)$ & $-0.02(-2.58,2.54)$ & $0(-0.03,0.02)$ & $0.09(-0.85,1.03)$ & $-0.97(-4.20,2.25)$ \\
\hline \multicolumn{7}{|l|}{ Model 1d } \\
\hline \multicolumn{7}{|l|}{$\mathrm{WAZCT}^{\ddagger}$} \\
\hline Steady & Ref. & Ref. & Ref. & Ref. & Ref. & Ref. \\
\hline Rapid & $0.48(-0.30,1.26)$ & $0.41(-0.26,1.09)$ & $0.48(-1.40,2.36)$ & $0.01(-0.01,0.02)$ & $0.47(-0.23,1.17)$ & $1.54(-0.81,3.89)$ \\
\hline \multicolumn{7}{|l|}{$\mathrm{FF}^{\zeta}$} \\
\hline Low & Ref. & Ref. & Ref. & Ref. & Ref. & Ref. \\
\hline High & $-0.66(-1.47,0.16)$ & $-0.70(-1.38,-0.02)^{*}$ & $-1.84(-3.75,0.07)$ & $0(-0.02,0.01)$ & $-0.58(-1.29,0.13)$ & $-2.20(-4.57,0.17)$ \\
\hline
\end{tabular}




\begin{tabular}{|c|c|c|c|c|c|c|}
\hline WAZCT*FR & $0.63(-0.45,1.71)$ & $0.64(-0.28,1.55)$ & $2.16(-0.39,4.72)$ & $0.01(-0.02,0.03)$ & $0.62(-0.33,1.57)$ & $1.66(-1.53,4.85)$ \\
\hline
\end{tabular}

$\mathrm{BMI}=$ body mass index $; \mathrm{CI}=$ confidence interval; $\mathrm{EF}=$ enjoyment of food; $\mathrm{FF}=$ food fussiness; $\mathrm{FR}=$ food responsiveness; $\mathrm{SR}=$ satiety responsiveness; $\mathrm{WH} \mathrm{tR}=\mathrm{waist}$-to-height ratio; $\mathrm{WAZCT}=$

WAZ-change trajectories, i.e. trajectory for change of weight-for-age z-score.

${ }^{\psi}$ Adjusted children's height at four years old.

$\zeta$ Low scores present the values less than the median levels of each subscale and the high scores present the values more than the median levels of each subscale.

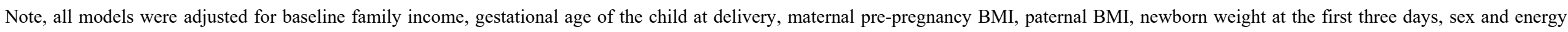
intake at six months.

$* P<0.05, * * P<0.01, * * * P<0.001$. 

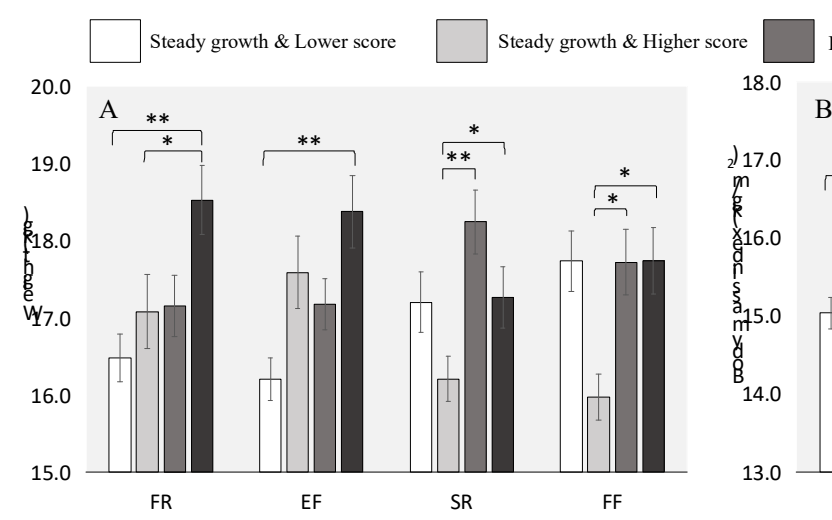

Rapid growth \& Lower score

Rapid growth \& Higher score

B

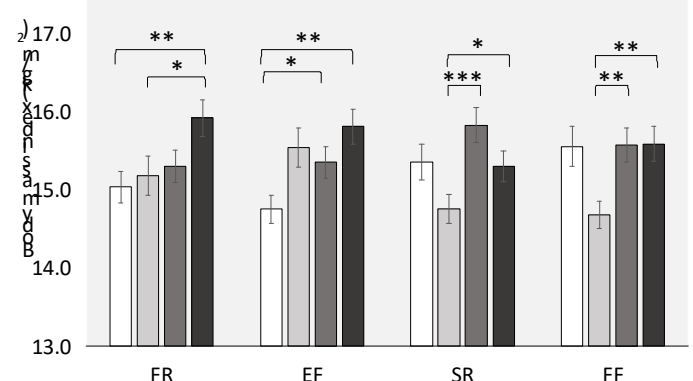

FR
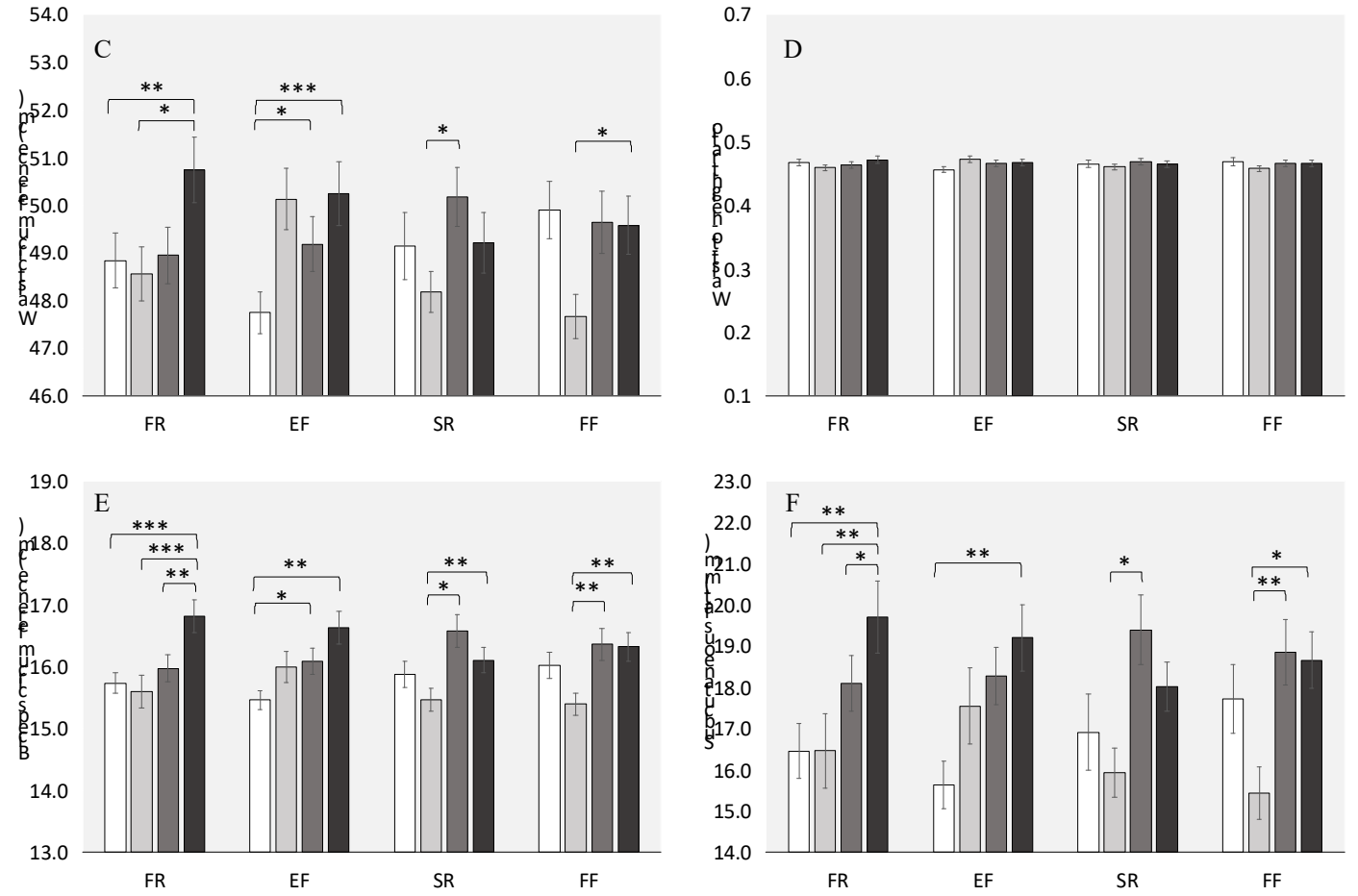

Figure S1 Combined effects of WAZ-change trajectories and four subscales of eating behaviors on children's adiposity measures at four years (non imputed data).

Note, EF indicates enjoyment of food; FF indicates food fussiness; FR indicates food responsiveness; SR indicates satiety responsiveness. Low scores present the values less than the median levels of each subscale and the high scores present the values more than the median levels of each subscale.

The Y-axes are: A, weight $(\mathrm{kg})$; B, body mass index $\left(\mathrm{kg} / \mathrm{m}^{2}\right)$; C, waist circumference $(\mathrm{cm})$; D, waist-to-height ratio; E, biceps circumference $(\mathrm{cm}) ; \mathrm{F}$, subcutaneous fat $(\mathrm{mm})$ respectively. The X-axes are the combined groups with the two distinct WAZ-change trajectories and the dichotomy variable of four subscales for eating behaviors (FR, EF, SR and FF). The white bars indicate children with steady growth trajectory and lower score of the four eating behaviors, the very light black bars indicate children with steady growth trajectory and higher score of the four eating behaviors, the moderate black bars indicate children with early infancy rapid growth trajectory and lower score of the four eating behaviors, and the dark black bars indicate children with early infancy rapid growth trajectory and higher score of the four eating behaviors. Note, EF indicates enjoyment of food; FF indicates food fussiness; FR indicates food responsiveness; SR indicates satiety responsiveness. Low scores present the values less than the median levels of each subscale and the high scores present the values more than the median levels of each subscale.

Error bars represent mean \pm s.e.m.

$* P<0.05, * * P<0.01,{ }^{* * *} P<0.001$. 